\title{
Noiseless subsystems and the structure of the commutant in quantum error correction
}

\author{
John A. Holbrook ${ }^{1}$, David W. Kribs ${ }^{1,3}$ and Raymond Laflamme ${ }^{2,3}$ \\ ${ }^{1}$ Department of Mathematics and Statistics, \\ University of Guelph, Guelph, Ontario, Canada N1G $2 W 1$. \\ ${ }^{2}$ Institute for Quantum Computing, University of Waterloo, \\ Waterloo, ON, CANADA N2L $3 G 1$. \\ ${ }^{3}$ Perimeter Institute for Theoretical Physics, \\ 35 King St. North, Waterloo, ON, CANADA N2J 2W9*
}

\begin{abstract}
The effect of noise on a quantum system can be described by a set of operators obtained from the interaction Hamiltonian. Recently it has been shown that generalized quantum error correcting codes can be derived by studying the algebra of this set of operators. This led to the discovery of noiseless subsystems. They are described by a set of operators obtained from the commutant of the noise generators. In this paper we derive a general method to compute the structure of this commutant in the case of unital noise.
\end{abstract}

*Electronic address: jholbroo@uoguelph.ca; dkribs@uoguelph.ca; laflamme@iqc.ca 


\section{INTRODUCTION}

Quantum mechanics promises to manipulate information for communication, cryptography and computation in a way fundamentally different from its classical counterpart [21]. Although it is possible to manipulate small quantum systems in the laboratory, the task to do so for large ones is daunting, especially because in absence of control of noise and imperfection of realistic devices the quantum properties of the state are destroyed. Quantum error correction methods have recently been discovered which protect quantum information against corruption. In particular it was shown that if the error rate is below a certain threshold [1, 15, 17, 24] then it is possible implement quantum error correction and obtain a fixed probability of success with only a polynomial amount of resources. The work on the accuracy threshold assumes certain error models such as independent or quasi independent errors. The theorem shows that under reasonable assumptions, imperfections and imprecisions of realistic devices do not create fundamental objections to scalable quantum information processing.

The estimated value of the error threshold is small and, today, an extraordinary challenge for experimentalists. From the experimental point of view there is a need to better understand the details of error models of quantum devices, the form of the Kraus noise operators which occur during the evolution of a quantum computation or during the transmission in a quantum channel, the correlation between errors, their strength, etc. From the theoretical side we need to find efficient ways to estimate the error model, determine the threshold for various physically relevant error models and find ways to achieve better threshold. This can be done by optimizing quantum error correction procedures or simply finding better ways to protect quantum information.

Recently a unified view of error correction was given which brought together quantum error correcting codes and noiseless (decoherence-free) subspaces [8, 16, 20, 29, 30]. This investigation led to the discovery of noiseless subsystems. A quantum bit, called a qubit, is described by a unit vector in a 2-dimensional Hilbert space. Often this 2-dimensional space is directly associated with a two-level physical system such as the ground and excited states of an atom, a nuclear spin pointing up or down, etc. Noiseless subsystems are instead described by a set of operators such as the Pauli matrices, a more general concept than

a subspace of a Hilbert space 16]. An example where the difference can be appreciated is 
in cases where the systems at hand are subject to noise with some symmetries and we are interested in a fraction of the operators acting on the whole Hilbert space of the systems. For example, the polarization of photons moving in an optic fiber undergo collective rotation. When a symmetry of the noise exists, it is possible to find conserved quantities and thus invariant, or fixed, operators. These operators form the noise commutant $\mathcal{A}^{\prime}$ of the noise.

When the channel determined by the transmission is unital (i.e. the unit matrix is preserved), the commutant is a $\mathrm{C}^{*}$-algebra 19]. Such an algebra has a block diagonal matrix form

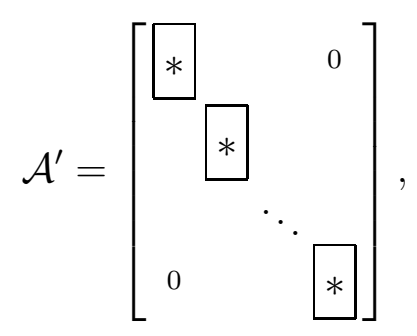

where the compression to each block forms a full matrix algebra. But some of these blocks may be 'linked' in a sense that we make precise below. Once the block matrix form and the various links between blocks have been found, the explicit form of the algebra is revealed. In particular, there are unique positive integers $n_{k}, m_{k} \geq 1$ for $k=1, \ldots, d$ such that the algebra is unitarily (spatially) equivalent to an orthogonal direct sum

$$
\mathcal{A}^{\prime} \simeq\left(\mathcal{M}_{n_{1}} \otimes \mathbb{1}_{m_{1}}\right) \oplus \ldots \oplus\left(\mathcal{M}_{n_{d}} \otimes \mathbb{1}_{m_{d}}\right),
$$

where $\mathcal{M}_{n_{k}}$ is the full $n_{k} \times n_{k}$ matrix algebra on $n_{k}$-dimensional Hilbert space and $\mathbb{1}_{m_{k}}$ is the identity operator on $m_{k}$-dimensional space. A tensor product $\mathcal{M}_{n_{k}} \otimes \mathbb{1}_{m_{k}}$ in this decomposition corresponds to $m_{k}$ blocks of $n_{k} \times n_{k}$ matrices in (1D) which are linked, and is referred to as an ampliation of $\mathcal{M}_{n_{k}}$ when $m_{k} \geq 2$. From the representation theory perspective, the integers $m_{k}$ correspond to the multiplicities of the identity representations of $\mathcal{M}_{n_{k}}$ which determine the structure of $\mathcal{A}^{\prime}$.

If there are invariant operators which can be decomposed into projectors of rank one, so they are supported on blocks with $n_{k}=1$, then they correspond to invariant subspaces and the usual notion of qubits. Otherwise the invariant operators correspond to noiseless subsystems on higher dimensional spaces. This can be read off the structure of the commutant. For instance, in the above discussion there will be a copy of every operator from $\mathcal{M}_{n_{k}}$ contained in the $\mathcal{M}_{n_{k}} \otimes \mathbb{1}_{m_{k}}$ component of the decomposition. Thus the structure of 
the commutant indicates how quantum information can be encoded to be preserved under the influence of the noise.

In this paper we study how to find the structure of the commutant once the noise (Kraus) operators are known. A constructive proof of this structure is given in the case of unital evolution. The paper is structured as follows. In sections 2 and 3 we review background concepts. In particular, we recall basic properties of quantum channels and results from [19] on the fixed point set of a unital channel. Section 4 contains the core theoretical component of the paper. Based on operator theory and operator algebra methods, we present a detailed algorithm for computing the noise commutant of a unital channel. We apply this method in Section 5 to find the commutant for a number of simple illustrative examples which are related to the phase damping channel [11, 14, 21]. Section 6 contains a short discussion on how to turn a non-unital channel into a unital one, when the noise operators satisfy certain constraints. In the final three sections we conduct an analysis of special cases of collective noise channels which arise from collective rotation [9, 10, 14, 16, 26, 27, 29]. Specifically, section 7 includes an introductory discussion on this class, and in the final two sections we use the algorithm to explicitly compute the commutant for the 3-qubit [10, 14, 26, 27] and 4-qubit channels from this class.

\section{CHANNEL PRELIMINARIES}

Let $\mathcal{H}$ be a finite dimensional Hilbert space and let $\mathcal{B}(\mathcal{H})$ be the set of all bounded (continuous) operators acting on $\mathcal{H}$. A quantum channel (or quantum evolution) on $\mathcal{H}$ is a linear map $\Phi: \mathcal{B}(\mathcal{H}) \rightarrow \mathcal{B}(\mathcal{H})$ which is completely positive and trace preserving. We mention the texts [22, 23] for basic properties of completely positive maps, and [21] for an introduction to channels in quantum information theory.

It is well-known [4, 18] that to every completely positive map $\Phi$ there corresponds a (non-unique) family of operators $\left\{A_{1}, \ldots, A_{n}\right\}$ in $\mathcal{B}(\mathcal{H})$ which determine the map $\Phi$ through the equation

$$
\Phi(T)=\sum_{k=1}^{n} A_{k} T A_{k}^{\dagger} \quad \text { for } \quad T \in \mathcal{B}(\mathcal{H})
$$


Trace preservation of $\Phi$ is equivalent to these operators satisfying

$$
\sum_{k=1}^{n} A_{k}^{\dagger} A_{k}=1
$$

where $\mathbb{1}$ is the identity operator on $\mathcal{H}$. (This is also equivalent to the 'dual map' of $\Phi$ being unital.) We say $\Phi$ is unital if also,

$$
\Phi(\mathbb{1})=\sum_{k=1}^{n} A_{k} A_{k}^{\dagger}=\mathbb{1} .
$$

For a quantum channel $\Phi$, the family of operators $\left\{A_{k}\right\}$ determining $\Phi$ as in (2) are called the Kraus noise operators for the channel.

The fixed point set for $\Phi$ will be denoted by

$$
\operatorname{Fix}(\Phi)=\{T \in \mathcal{B}(\mathcal{H}): \Phi(T)=T\}
$$

We shall also let $\mathcal{A}$ be the so-called interaction algebra [16] generated by the noise operators,

$$
\mathcal{A}=\operatorname{Alg}\left\{A_{1}, \ldots, A_{n}\right\}
$$

In other words, $\mathcal{A}$ is the set of all polynomials in the typically noncommuting variables $A_{1}, \ldots, A_{n}$. Note that as an application of the Cayley-Hamilton theorem from linear algebra, the degree of any such polynomial may be reduced below some uniform bound. The noise commutant $\mathcal{A}^{\prime}$ of $\mathcal{A}$ is the set of all operators which commute with every operator in $\mathcal{A}$; it is the algebra

$$
\begin{aligned}
\mathcal{A}^{\prime} & =\{T \in \mathcal{B}(\mathcal{H}): T A=A T \quad \text { for all } \quad A \in \mathcal{A}\} \\
& =\left\{T \in \mathcal{B}(\mathcal{H}): T A_{k}=A_{k} T \text { for } \quad k=1, \ldots, n\right\}
\end{aligned}
$$

Remark II.1 The interaction algebra, and hence the noise commutant, are usually defined as $\dagger$-algebras [16]. However, in the case considered here, that of unital noise, the discussion below shows that our 'nonselfadjoint' definitions are actually a red herring, the algebras turn out to be selfadjoint.

In this paper, we shall focus our attention on unital quantum channels. These are sometimes called bistochastic channels in the literature. For such channels, it was proved in [19] that: 
(i) $\mathcal{A}$ is a $\dagger$-algebra; in other words, $A$ belongs to $\mathcal{A}$ if and only if $A^{\dagger}$ belongs to $\mathcal{A}$. Furthermore, the algebra $\mathcal{A}$ is independent of the choice of noise operators which determine the channel as in (2); the same algebra is obtained whatever the choice of operators $\left\{A_{k}\right\}$.

(ii) The commutant $\mathcal{A}^{\prime}$ is a $†$-algebra which is equal to the fixed point set of $\Phi$,

$$
\mathcal{A}^{\prime}=\operatorname{Fix}(\Phi)
$$

These results are recalled in the next section. Taken together, they may be regarded as a structure theorem for the fixed point set of a unital quantum channel. In particular, the fixed point set $\operatorname{Fix}(\Phi)$ (which is just a $†$-closed subspace for general completely positive maps) is itself an algebra, hence closed under multiplication, equal to the noise commutant. But there is a tight decomposition theory for finite dimensional †-algebras (see section IV] for details). This motivates the following fundamental problem which we consider in this paper:

Problem II.2 Given a unital quantum channel $\Phi$, explicitly determine the algebra structure of the noise commutant $\operatorname{Fix}(\Phi)=\mathcal{A}^{\prime}$.

There are compelling reasons in quantum information theory for wishing to solve this problem. For instance, in the noiseless subsystem method of quantum error correction [8, 10, 16, 20, 27, 30], the structure of the noise commutant can be used to encode quantum information which is immune to the noise of the corresponding channel.

\section{STRUCTURE THEOREM}

In 19] it was discovered that many properties of a channel are married to properties of the noise operators. Recall that an orthogonal projection $P$ on $\mathcal{H}$ reduces an operator $T$ in $\mathcal{B}(\mathcal{H})$ when both subspaces $P \mathcal{H}$ and $(\mathbb{1}-P) \mathcal{H}=P^{\perp} \mathcal{H}$ are invariant for $T$; that is, $T(P \mathcal{H}) \subseteq P \mathcal{H}$ and $T\left(P^{\perp} \mathcal{H}\right) \subseteq P^{\perp} \mathcal{H}$. This is equivalent to the identity $T P=P T$ being satisfied, or $T$ having a block diagonal matrix form $T=\left[\begin{array}{cc}B & 0 \\ 0 & C\end{array}\right]$ with respect to the orthogonal spatial decomposition $\mathcal{H}=P \mathcal{H} \oplus P^{\perp} \mathcal{H}$. Further recall the operator inequality $P \leq Q$ for a pair of self-adjoint operators on $\mathcal{H}$ means that $\langle P \xi \| \xi\rangle \leq\langle Q \xi \| \xi\rangle$ for all $\xi \in \mathcal{H}$. The key 
point in the following result is that invariant subspaces for the noise operators of a unital channel are always reducing subspaces.

Lemma III.1 [19] Let $\Phi: \mathcal{B}(\mathcal{H}) \rightarrow \mathcal{B}(\mathcal{H})$ be a unital completely positive map and let $P$ be a projection on $\mathcal{H}$. Consider the following conditions.

(i) $\Phi(P)=P$.

(ii) The subspace $P \mathcal{H}$ reduces $A_{1}, \ldots, A_{n}$;

$$
A_{k} P=P A_{k} \quad \text { for } \quad 1 \leq k \leq n .
$$

(iii) $\Phi(P) \leq P$.

(iv) The subspace $P \mathcal{H}$ is invariant for $A_{1}, \ldots, A_{n}$;

$$
A_{k} P=P A_{k} P \quad \text { for } \quad 1 \leq k \leq n .
$$

(v) $\Phi(P) \geq P$.

(vi) The subspace $P \mathcal{H}$ is invariant for $A_{1}^{\dagger}, \ldots, A_{n}^{\dagger}$;

$$
P A_{k}=P A_{k} P \quad \text { for } \quad 1 \leq k \leq n .
$$

Then the pairs of conditions $((i),(i i)),((i i i),(i v))$, and $((v),(v i))$ are each equivalent. Moreover, if $\Phi$ is a unital channel then all six conditions are equivalent to each other. Further, given a projection $P$ and a unital channel $\Phi$, conditions $(i i),(i v)$ and (vi) hold for one choice of noise operators for $\Phi$ if and only if they hold for all choices.

Let us outline the proof of $(i v) \Rightarrow(i i)$ for a unital channel $\Phi$, since this is the aspect of Lemma III.1 used in the proof of Theorem ஹI.4. Suppose $(i v)$ holds for a projection $P$ in $\mathcal{B}(\mathcal{H})$. Then

$$
0 \leq \Phi(P)=\sum_{i} A_{i} P A_{i}^{\dagger}=P \Phi(P) P \leq P \Phi(\mathbb{1}) P=P \mathbb{1} P=P
$$

As $0 \leq \Phi(P) \leq P$, trace preservation can be seen to imply through an operator theory argument that $\Phi(P)=P$. More generally, if $R$ is a positive contraction operator with 
$R \leq P$ for some projection $P$ and $R$ has trace equal to the trace of $P$, then in fact $R=P$. Thus $\Phi\left(P^{\perp}\right)=\Phi(\mathbb{1}-P)=P^{\perp}$ since $\Phi$ is unital, and for each $j$

$$
\begin{aligned}
0 & \leq\left(P^{\perp} A_{j}^{\dagger} P\right)^{\dagger}\left(P^{\perp} A_{j}^{\dagger} P\right) \\
& \leq \sum_{i}\left(P^{\perp} A_{i}^{\dagger} P\right)^{\dagger}\left(P^{\perp} A_{i}^{\dagger} P\right)=P \Phi\left(P^{\perp}\right) P=P P^{\perp} P=0 .
\end{aligned}
$$

Thus, $P^{\perp} A_{j}^{\dagger} P=0$ for all $j$, and hence $P \mathcal{H}$ is a reducing subspace for $A_{1}, \ldots, A_{n}$.

We mention that this lemma gives a simple characterization of rank one projections fixed by a unital channel.

Corollary III.2 Let $P=|\xi\rangle\langle\xi|$ be a rank one projection in $\mathcal{B}(\mathcal{H})$. Then the following are equivalent:

(i) $\Phi(P)=P$.

(ii) The range vector $\xi$ for $P$ is a joint eigenvector for the noise operators $A_{1}, \ldots, A_{n}$;

$$
A_{k} \xi=\lambda_{k} \xi \quad \text { for some } \quad \lambda_{k} \in \mathbb{C} \quad \text { and } \quad k=1, \ldots, n .
$$

Proof. Condition (ii) here is simply a restatement of $(i v)$ from the lemma, in the special case of a rank one projection.

We recall some terminology from linear algebra before proceeding.

Definition III.3 Let $T$ be a normal operator in $\mathcal{B}(\mathcal{H})$, in other words $T T^{\dagger}=T^{\dagger} T$. Then a spectral projection for $T$ is a projection onto an eigenspace for $T$; that is, it is a projection onto a subspace of the form $\{\xi \in \mathcal{H}: T \xi=\lambda \xi\}$ for some $\lambda \in \mathbb{C}$. The Spectral Theorem from linear algebra states that every normal operator $T$ decomposes as a sum $T=\lambda_{1} P_{1}+\ldots+\lambda_{r} P_{r}$, where $\lambda_{i} \in \mathbb{C}$ are the distinct eigenvalues for $T$ and $P_{i}$ the corresponding spectral projections (which necessarily have mutually orthogonal ranges).

Clearly the fixed point set $\operatorname{Fix}(\Phi)$ contains the noise commutant $\mathcal{A}^{\prime}$; if $T$ belongs to $\mathcal{A}^{\prime}$ then

$$
\Phi(T)=\sum_{k=1}^{n} A_{k} T A_{k}^{\dagger}=T\left(\sum_{k=1}^{n} A_{k} A_{k}^{\dagger}\right)=T \Phi(\mathbb{1})=T .
$$

The converse inclusion holds for unital channels. We mention that the following theorem was partly motivated by work from [6] on infinite dimensional operator algebras and dilation theory. The proof in [19] is somewhat technical, but after submission we discovered a simpler proof of this fact, and hence, for completeness, we shall present it here. 
Theorem III.4 [19] Let $\Phi: \mathcal{B}(\mathcal{H}) \rightarrow \mathcal{B}(\mathcal{H})$ be a unital quantum channel determined as in (2) by operators $\left\{A_{1}, \ldots, A_{n}\right\}$. Then the fixed point set for $\Phi$ is a $\dagger$-algebra and is equal to the noise commutant,

$$
\operatorname{Fix}(\Phi)=\mathcal{A}^{\prime}=\left\{A_{1}, \ldots, A_{n}\right\}^{\prime}
$$

Further, the interaction algebra $\mathcal{A}$ generated by $A_{1}, \ldots, A_{n}$ is a †-algebra which depends only on $\Phi$ via the equation

$$
\mathcal{A}=\operatorname{Fix}(\Phi)^{\prime}
$$

Proof. Notice that the theorem will be proved if it is shown that $\mathcal{A}^{\prime}=\operatorname{Fix}(\Phi)$. Indeed, $\mathcal{A}^{\prime}$ is an algebra by definition and $\operatorname{Fix}(\Phi)$ is $\dagger$-closed since $\Phi$ is a positive map. Moreover, if this identity holds, the algebra $\mathcal{A}$ depends only on $\Phi$, not the choice of noise operators, since $\mathcal{A}=\left(\mathcal{A}^{\prime}\right)^{\prime}=\operatorname{Fix}(\Phi)^{\prime}$. (This is a special case of von Neumann's double commutant theorem from operator algebras.)

As observed above, the commutant $\mathcal{A}^{\prime}$ is contained in $\operatorname{Fix}(\Phi)$ because $\Phi$ is unital. To see the converse, first observe that if the only fixed points are scalars, then $\mathbb{C} \mathbb{1} \subseteq \mathcal{A}^{\prime} \subseteq \operatorname{Fix}(\Phi)=$ $\mathbb{C} 1$, and the two sets coincide. Otherwise, let $T$ be a non-scalar operator in $\operatorname{Fix}(\Phi)$. Without loss of generality we may assume that $T$ is self-adjoint; in other words, $T=T^{\dagger}$. Indeed, $\Phi\left(T^{\dagger}\right)=\Phi(T)^{\dagger}=T^{\dagger}$ by positivity of $\Phi$, and hence both the real, $\operatorname{Re}(T)=\frac{1}{2}\left(T+T^{\dagger}\right)$, and imaginary, $\operatorname{Im}(T)=\frac{1}{2 i}\left(T-T^{\dagger}\right)$, parts of $T=\operatorname{Re}(T)+i \operatorname{Im}(T)$ are fixed by $\Phi$. Since $T$ is non-scalar, at least one of these self-adjoint operators is non-scalar. Furthermore, by replacing $T$ with $T+\|T\| \mathbb{1}$, we may assume $T \geq 0$ is a positive operator. Let $0 \leq \lambda_{1}<\lambda_{2}<$ $\ldots<\lambda_{r}$ be the eigenvalues for $T$, and $P_{1}, \ldots, P_{r}$ the corresponding spectral projections. Then $T=\lambda_{1} P_{1}+\ldots+\lambda_{r} P_{r}$ by the Spectral Theorem, and $0 \leq T \leq\|T\| \mathbb{1}=\lambda_{r} \mathbb{1}$. Let $\mathcal{H}_{r}=P_{r} \mathcal{H}=\left\{\xi \in \mathcal{H}: T \xi=\lambda_{r} \xi\right\}$ be the eigenspace for the extremal eigenvalue $\lambda_{r}$. If $\xi$ is a non-zero vector in $\mathcal{H}_{r}$, observe that

$$
\begin{aligned}
\lambda_{r}\langle\xi \| \xi\rangle=\langle T \xi \| \xi\rangle & =\sum_{k=1}^{n}\left\langle A_{k} T A_{k}^{\dagger} \xi \| \xi\right\rangle \\
& =\sum_{k=1}^{n}\left\langle T A_{k}^{\dagger} \xi \| A_{k}^{\dagger} \xi\right\rangle \\
& \leq \lambda_{r} \sum_{k=1}^{n}\left\langle A_{k}^{\dagger} \xi \| A_{k}^{\dagger} \xi\right\rangle=\lambda_{r}\langle\xi \| \xi\rangle .
\end{aligned}
$$

The only way this can happen is if each of the inequalities is actually an equality; in particular, $A_{k}^{\dagger} \xi$ belongs to $\mathcal{H}_{r}$ for $k=1, \ldots, n$. Thus $\mathcal{H}_{r}=P_{r} \mathcal{H}$ is an invariant subspace for 
$\mathcal{A}^{\dagger}$, and so it is a reducing subspace for $\mathcal{A}$ by Lemma ஹI.1. Therefore, it follows that $P_{r}$ belongs to the commutant $\mathcal{A}^{\prime}$, and hence to the fixed point set $\operatorname{Fix}(\Phi)$. Thus the self-adjoint operator $T-\lambda_{r} P_{r}$ belongs to $\operatorname{Fix}(\Phi)$, and we may iterate this argument to find that each of $P_{1}, \ldots, P_{r}$ belongs to $\mathcal{A}^{\prime}$. Hence $T$ belongs to $\mathcal{A}^{\prime}$, as required.

We have shown that every $T=T^{\dagger}$ in $\operatorname{Fix}(\Phi)$ also belongs to $\mathcal{A}^{\prime}$. But $\operatorname{Fix}(\Phi)$ is a selfadjoint subspace, and hence spanned by its self-adjoint part. It follows that the fixed point set $\operatorname{Fix}(\Phi)$ is contained in the commutant $\mathcal{A}^{\prime}$, and this completes the proof.

\section{COMPUTING THE COMMUTANT}

In this section, using operator theory and operator algebra techniques, we present a constructive process for explicitly computing the noise commutant $\mathcal{A}^{\prime}$ for a unital quantum channel. This will be accomplished by first identifying the $\dagger$-algebra structure of the interaction algebra $\mathcal{A}$. We begin with a short discussion on the decomposition theory for $\dagger$-algebras.

A fundamental result from the theory of operator algebras is that every $\dagger$-algebra $\mathcal{A}$ is $\dagger$-isomorphic (an isomorphism which preserves adjoints) to a unique orthogonal direct sum of full matrix algebras. Specifically, there are positive integers $n_{k} \geq 1$ such that $\mathcal{A}$ is $\dagger$-isomorphic to

$$
\mathcal{A} \simeq \mathcal{M}_{n_{1}} \oplus \ldots \oplus \mathcal{M}_{n_{d}}
$$

We mention the texts [2, 5, 25] for basics of $\dagger$-algebras, or equivalently, finite dimensional $\mathrm{C}^{*}$-algebras. Another important result is that every representation of such an algebra decomposes into a direct sum of multiples of the identity representation on each summand. As a consequence, up to unitary equivalence $\mathcal{A}$ is given by a unique direct sum of 'ampliated' full matrix algebras,

$$
\mathcal{A} \simeq\left(\mathcal{M}_{n_{1}} \otimes \mathbb{1}_{m_{1}}\right) \oplus \ldots \oplus\left(\mathcal{M}_{n_{d}} \otimes \mathbb{1}_{m_{d}}\right) .
$$

In other words, there is a unitary operator $U$ such that $U^{\dagger} \mathcal{A} U$ is equal to the decomposition in (44). See also [10, 14, 26, 28, 29] for discussions of these decompositions in connection with quantum information processing. 
While it is important to know the $\dagger$-isomorphism class of $\mathcal{A}$ as in (3), we really need the structure of $\mathcal{A}$ spatially as in (41). More to the point, we need to identify the precise spatial locations of the matrix blocks $\mathcal{M}_{n_{k}} \otimes \mathbb{1}_{m_{k}}$. The integers $m_{k} \geq 1$ are the multiplicities of the identity representation appearing in each summand associated with the corresponding representation of the $\nmid$-isomorphism class model in (3). The invariants $n_{k}, m_{k}$ are part of what is known as the Wedderburn structure theory for such algebras. When some $m_{k} \geq$ 2, we say $\mathcal{M}_{n_{k}} \otimes \mathbb{1}_{m_{k}}$ is an ampliation of $\mathcal{M}_{n_{k}}$. The projections in $\mathcal{A}$ which, under the unitary equivalence (4), correspond to the identity operators on the blocks $\mathcal{M}_{n_{k}} \otimes \mathbb{1}_{m_{k}}$ in the decomposition (4) are called the minimal central projections for $\mathcal{A}$. Each of these projections will be a sum of projections onto some, but perhaps not all, of the matrix blocks of the same size in the block diagonal form (1).

The projections onto the individual matrix blocks in (1) will be minimal inside $\mathcal{A}^{\prime}$, in other words minimal $\mathcal{A}$-reducing, but may not belong to the algebra $\mathcal{A}$. This is precisely the subtlety we must deal with in this process; an identification of 'links' between these minimal projections. Thus, broadly speaking, we must first identify the maximal (here meaning that the projections sum to the identity operator) family of non-zero minimal $\mathcal{A}$-reducing projections with mutually orthogonal ranges (part I below); it is easy to see that such a family of projections is unique. Then we must detect any links between these projections (part II below) in the following sense.

Definition IV.1 Let $\left\{P_{j}\right\}$ be the (unique) maximal family of non-zero mutually orthogonal minimal $\mathcal{A}$-reducing projections. A subset $\left\{Q_{k}\right\}_{k \in \mathcal{S}}$ of this family is linked in $\mathcal{A}$ if the following two conditions hold.

(i) The projection $Q=\sum_{k \in \mathcal{S}} Q_{k}$ belongs to $\mathcal{A}$.

(ii) If $\mathcal{S}_{1}$ is a proper subset of $\mathcal{S}$, then the projection $\sum_{k \in \mathcal{S}_{1}} Q_{k}$ does not belong to $\mathcal{A}$.

Let $\left\{P_{j}: j \in \mathcal{S}_{k}\right\}_{k}$ be the linked subsets of projections from $\left\{P_{j}\right\}$. It follows that each algebra $\mathcal{A}\left(\sum_{j \in \mathcal{S}_{k}} \oplus P_{j}\right)$ forms a subalgebra of $\mathcal{A}$. Notice that, in terms of (4) , the cardinality of the sets $\mathcal{S}_{k}$ are equal to the ampliation multiplicities $m_{k}$.

Once the $\dagger$-algebra structure of $\mathcal{A}$ has been identified up to unitary equivalence, we can easily deduce the $\dagger$-algebra structure of $\mathcal{A}^{\prime}=\operatorname{Fix}(\Phi)$. For instance, if $\Phi$ is a channel and the 
spatial structure of $\mathcal{A}$ is given by (4), then

$$
\operatorname{Fix}(\Phi)=\mathcal{A}^{\prime} \simeq \sum_{k} \oplus\left(\mathcal{M}_{n_{k}} \otimes \mathbb{1}_{m_{k}}\right)^{\prime} \simeq \sum_{k} \oplus\left(\mathbb{1}_{n_{k}} \otimes \mathcal{M}_{m_{k}}\right)
$$

Furthermore, the algorithm presented below will allow us to explicitly identify the locations of the matrix blocks, possibly ampliated, which belong to the noise commutant.

We shall work our way through the finer points of the process, then finish by summarizing the main points. For the rest of this section we let $\Phi$ be a given unital quantum channel acting on $\mathcal{B}(\mathcal{H})$, with noise operators $\left\{A_{1}, \ldots, A_{n}\right\}$, and interaction algebra $\mathcal{A}=\operatorname{Alg}\left\{A_{1}, \ldots, A_{n}\right\}$. The following assumption gives us the starting point.

\section{Assumption: The noise commutant $\mathcal{A}^{\prime}=\operatorname{Fix}(\Phi)$ has been computed as a linear span.}

The algorithm will take us from knowing the vector space structure of $\mathcal{A}^{\prime}$, to identifying the algebra structure of $\mathcal{A}$, and hence of $\mathcal{A}^{\prime}$. There are two broad components to the process:

Part I. Find the family of non-zero projections inside the noise commutant $\mathcal{A}^{\prime}$ which have mutually orthogonal ranges, sum to the identity operator, and are each minimal inside $\mathcal{A}^{\prime}$.

Part II. Given the family of projections $\left\{P_{j}\right\}$ from Part I, find the minimal central projections for $\mathcal{A}$ by computing all links between the $P_{j}$. This will give the spatial $\dagger$-algebra structure of $\mathcal{A}$, and hence of $\operatorname{Fix}(\Phi)=\mathcal{A}^{\prime}$.

\section{A. Part I}

As $\Phi$ is unital, $\mathcal{A}^{\prime}=\operatorname{Fix}(\Phi)$ always contains the scalar operators. If $\mathcal{A}^{\prime}=\mathbb{C} 1$, then the singleton $\{\mathbb{1}\}$ is the only (non-zero) minimal central projection for $\mathcal{A}$. Otherwise, let $T=T^{\dagger}$ belong to $\mathcal{A}^{\prime} \backslash \mathbb{C} 1$. The spectral projections for $T$ provide the first step toward the maximal family.

Lemma IV.2 The following are equivalent for an operator $T=T^{\dagger}$ in $\mathcal{B}(\mathcal{H})$ :

(i) $\Phi(T)=T$.

(ii) Every spectral projection $P$ of $T$ satisfies $\Phi(P)=P$. 
(iii) Every spectral projection $P$ of $T$ belongs to $\mathcal{A}^{\prime}$.

Proof. Condition (ii) and (iii) are equivalent by Theorem III.4. The equivalence of the first two conditions immediately follows from standard operator algebra for $\uparrow$-algebras. We provide a proof for the sake of brevity. The implication $(i i) \Rightarrow(i)$ follows from the Spectral Theorem of linear algebra; $T$ decomposes as a sum $T=\lambda_{1} P_{1}+\ldots+\lambda_{r} P_{r}$ where $\lambda_{1}, \ldots, \lambda_{r}$ are the distinct eigenvalues for $T$, and $P_{1}, \ldots, P_{r}$ the corresponding spectral projections. On the other hand, if $\Phi(T)=T$, then $T$ belongs to the commutant $\mathcal{A}^{\prime}$ by Theorem 111.4 But again by the Spectral Theorem, the spectral projections of $T$ commute with everything that commutes with $T$. Hence $(i) \Rightarrow(i i)$ follows since $\mathcal{A}^{\prime}=\operatorname{Fix}(\Phi)$.

Thus, if we let $\left\{P_{j}\right\}$ be the (mutually orthogonal) spectral projections of $T$, then $P_{j}$ belongs to $\operatorname{Fix}(\Phi)=\mathcal{A}^{\prime}$ for each $j$. Observe that for all $j$ we may define a map $\Phi_{j}$ : $\mathcal{B}\left(P_{j} \mathcal{H}\right) \rightarrow \mathcal{B}\left(P_{j} \mathcal{H}\right)$ by

$$
\Phi_{j}(S)=\sum_{k=1}^{n} A_{k, j} S A_{k, j}^{\dagger}
$$

where

$$
A_{k, j} \equiv A_{k} P_{j}=P_{j} A_{k}=P_{j} A_{k} P_{j} .
$$

It should be kept in mind that this is a slight abuse of notation; we should really write $A_{k, j}$ as the restriction $A_{k, j}=\left.A_{k}\right|_{P_{j} \mathcal{H}}$. These are unital channels since

$$
\Phi_{j}\left(\mathbb{1}_{P_{j} \mathcal{H}}\right)=\sum_{k=1}^{n} A_{k, j} A_{k, j}^{\dagger}=\sum_{k=1}^{n} P_{j} A_{k} A_{k}^{\dagger} P_{j}=P_{j} \Phi\left(\mathbb{1}_{\mathcal{H}}\right) P_{j}=\mathbb{1}_{P_{j} \mathcal{H}}
$$

A similar argument can be used to verify trace preservation for $\Phi_{j}$; that is, $\sum_{k=1}^{n} A_{k, j}^{\dagger} A_{k, j}=$ $\mathbb{1}_{P_{j} \mathcal{H}}$. Proposition $1 \nabla .4$ below uses an analysis of these maps to give a test for deciding if $P_{j}$ is minimal.

Lemma IV.3 Let $\Psi: \mathcal{B}(\mathcal{H}) \rightarrow \mathcal{B}(\mathcal{H})$ be a unital quantum channel, with noise operators $C_{1}, \ldots, C_{m}$, and let $\mathcal{C}=\operatorname{Alg}\left\{C_{1}, \ldots, C_{m}\right\}$. The following are equivalent:

(i) $\operatorname{Fix}(\Psi)=\mathcal{C}^{\prime}=\mathbb{C}_{\mathcal{H}}$.

(ii) There are no non-trivial reducing subspaces for the algebra $\mathcal{C}$.

Proof. This immediately follows from Theorem [II.4 since $\operatorname{Fix}(\Psi)=\mathcal{C}^{\prime}$ and $\mathcal{C}$ is a $†$-algebra. 
Proposition IV.4 Let $\Psi: \mathcal{B}(\mathcal{H}) \rightarrow \mathcal{B}(\mathcal{H})$ be a unital channel with noise operators $\left\{C_{i}\right\}$, and let $P \in \mathcal{B}(\mathcal{H})$ be a projection which reduces the noise operator algebra $\mathcal{C}$ for $\Psi$. Then the unital channel $\Psi_{P}: \mathcal{B}(P \mathcal{H}) \rightarrow \mathcal{B}(P \mathcal{H})$ given by $\Psi_{P}(S)=\sum_{i} C_{i, P} S C_{i, P}^{\dagger}$, where $C_{i, P}=$ $C_{i} P=P C_{i}$, satisfies

$$
\operatorname{Fix}\left(\Psi_{P}\right)=(\mathcal{C} P)^{\prime}=P \mathcal{C}^{\prime} P
$$

Furthermore, $P$ is a minimal projection in $\mathcal{C}^{\prime}$ if and only if

$$
\operatorname{Fix}\left(\Psi_{P}\right)=P \mathcal{C}^{\prime} P=\mathbb{C} P
$$

Proof. Again, the statement $(\mathcal{C} P)^{\prime}=P \mathcal{C}^{\prime} P$ is a notational convenience. We really mean that the compressed commutant, $\left.P \mathcal{C}^{\prime}\right|_{P \mathcal{H}}$, is equal to the commutant of the restriction, $\left(\left.\mathcal{C}\right|_{P \mathcal{H}}\right)^{\prime}=\left(\left.P \mathcal{C}\right|_{P \mathcal{H}}\right)^{\prime}$, inside $\mathcal{B}(P \mathcal{H})$. To see this, first let $T$ belong to $\mathcal{C}^{\prime}$. Then

$$
\begin{aligned}
(P T P)\left(C_{i} P\right) & =P\left(T C_{i}\right) P=P\left(C_{i} T\right) P \\
& =\left(P C_{i} P\right)(P T P)=\left(C_{i} P\right)(P T P),
\end{aligned}
$$

so that $(\mathcal{C} P)^{\prime}$ contains $P \mathcal{C}^{\prime} P$. Conversely, if $T=P T P$ is in $(\mathcal{C} P)^{\prime}$, then

$$
T C_{i}=T P C_{i}=T\left(C_{i} P\right)=\left(C_{i} P\right) T=C_{i} T,
$$

and $T$ belongs to $P \mathcal{C}^{\prime} P$. Thus Theorem $\llbracket .4$ gives us

$$
\operatorname{Fix}\left(\Psi_{P}\right)=\left\{C_{i, P}\right\}^{\prime}=\left\{C_{i} P\right\}^{\prime}=(\mathcal{C} P)^{\prime}=P \mathcal{C}^{\prime} P .
$$

The rest of the result follows from the previous lemma.

If we apply this result for $P=P_{j}$, we see that $P_{j}$ is a minimal projection inside $\mathcal{A}^{\prime}$ if and only if $P_{j} \mathcal{A}^{\prime} P_{j}=\mathbb{C} P_{j}$. Thus, since we are assuming the noise commutant $\mathcal{A}^{\prime}$ is known as a linear span, we can simply compute $P_{j} B P_{j}$ for a set of vector space generators $B$ for $\mathcal{A}^{\prime}$ and check if each $P_{j} B P_{j}$ belongs to $\mathbb{C} P_{j}$. If this is the case, then $P_{j}$ is minimal and it belongs to the maximal family of projections we seek. On the other hand, if there is a $B_{0}$ in $\mathcal{A}^{\prime}$ such that $P_{j} B_{0} P_{j}$ is not in $\mathbb{C} P_{j}$, then $P_{j}$ is not minimal; however, in this case we may iterate the process outlined above. Indeed, $\Phi_{j}$ is a unital channel on $\mathcal{B}\left(P_{j} \mathcal{H}\right)$ with nonscalar fixed points, in fact $P_{j} B_{0} P_{j} \in \mathcal{A}^{\prime} P_{j}=\operatorname{Fix}\left(\Phi_{j}\right)$ is such an operator. Thus, we may use $P_{j} B_{0} P_{j}$ to obtain a self-adjoint operator in $\operatorname{Fix}\left(\Phi_{j}\right)$, and continue the process by examining the spectral projections of this operator, which are, of course, each a subprojection of $P_{j}$. 
As $\mathcal{H}$ is finite dimensional, this process will eventually terminate. We will be left with the maximal family (since they sum to the identity operator) of mutually orthogonal non-zero minimal projections in $\mathcal{A}^{\prime}$, and this completes Part I.

\section{B. Part II}

We emphasize the importance of distinguishing the various links between projections in the maximal family from Part I by presenting the following simple example.

Example IV.5 The point is that we must distinguish between bonafide orthogonal direct sums on the one hand and ampliation algebras on the other by finding linked projections. Let

$$
\mathcal{C}=\left\{\left(\begin{array}{ll}
a & 0 \\
0 & a
\end{array}\right): a \in \mathbb{C}\right\} \simeq \mathbb{C} \mathbb{1}_{2} \simeq \mathbb{C} \otimes \mathbb{1}_{2},
$$

be a scalar ampliation algebra and let $\mathcal{D}$ be the unlinked orthogonal direct sum given by

$$
\mathcal{D}=\left\{\left(\begin{array}{ll}
a & 0 \\
0 & b
\end{array}\right): a, b \in \mathbb{C}\right\} \simeq \mathbb{C} \mathbf{1} \oplus \mathbb{C} \mathbf{1},
$$

where both of these $\uparrow$-algebras are diagonal with respect to the orthonormal basis $\{|0\rangle,|1\rangle\}$ for $\mathbb{C}^{2}$.

The family $\{|0\rangle\langle 0|| 1\rangle,\langle 1|\}$ of rank one projections is the maximal family from Part I for both algebras. However, the commutant of the scalar ampliation algebra $\mathcal{C}=\mathbb{C} \mathbb{1}_{2}$ is unitarily equivalent to $\mathcal{C}^{\prime} \simeq \mathcal{M}_{2}$, whereas the commutant of the orthogonal direct sum $\mathcal{D} \simeq \mathbb{C} 1 \oplus \mathbb{C} 1$ is also $\mathcal{D}^{\prime} \simeq \mathbb{C} \mathbf{1} \oplus \mathbb{C} 1$, the direct sum of two copies of the one dimensional scalar algebra. Notice that the projections $|0\rangle\langle 0|$ and $|1\rangle\langle 1|$ are linked in $\mathcal{C}$, since $\mathcal{C}$ contains $\mathbb{1}_{2}=|0\rangle\langle 0|+| 1\rangle\langle 1|$ and neither of these subprojections belongs to $\mathcal{C}$. On the other hand, they are unlinked in $\mathcal{D}$ because both projections already belong to $\mathcal{D}$.

Before continuing, let us recall a helpful identity from operator algebras. As a $\uparrow$-algebra, $\mathcal{A}$ is equal to its own double commutant, $\mathcal{A}=\left(\mathcal{A}^{\prime}\right)^{\prime}=\mathcal{A}^{\prime \prime}$. This is a special case of von Neumann's double commutant theorem [2, 5, 25]. Now, let $\left\{P_{j}\right\}$ be the family of projections for $\mathcal{A}$ obtained in Part I. It follows from the discussion at the start of this section that links can only occur between $P_{j}$ of the same rank. Hence for $k \geq 1$, let $\left\{P_{j, k}\right\}$ be the projections 
amongst $\left\{P_{j}\right\}$ with $\operatorname{dim} P_{j, k} \mathcal{H}=k$. Further let $\mathcal{S}_{k}$ be the index set for this set of projections. Suppose $\mathcal{A}^{\prime}=\operatorname{span}\left\{B_{1}, \ldots, B_{r}\right\}$ with $B_{i}$ in $\mathcal{B}(\mathcal{H})$.

Since $\mathcal{A}=\left(\mathcal{A}^{\prime}\right)^{\prime}=\left(\operatorname{span}\left\{B_{1}, \ldots, B_{r}\right\}\right)^{\prime}$, we may check if projections of the form $P_{\mathcal{S}}=$ $\sum_{j \in \mathcal{S}} P_{j, k}$, where $\mathcal{S} \subseteq \mathcal{S}_{k}$, belong to $\mathcal{A}$ simply by computing the commutators

$$
\left[P_{\mathcal{S}}, B_{i}\right]=P_{\mathcal{S}} B_{i}-B_{i} P_{\mathcal{S}} \quad \text { for } \quad i=1, \ldots, r
$$

The projections $\left\{P_{j, k}\right\}_{j \in \mathcal{S}}$ are linked in $\mathcal{A}$ precisely when $\left[P_{\mathcal{S}}, B_{i}\right]=0$ for each $i$, and, there is no proper subset $\mathcal{S}_{1}$ of $\mathcal{S}$ such that $\left[P_{\mathcal{S}_{1}}, B_{i}\right]=0$ for each $i$.As $\mathcal{H}$ is finite dimensional, there are just finitely many computations required here. Thus, we will eventually exhaust all possibilities and discover the links between the projections $\left\{P_{j, k}\right\}$.

Testing for links between the rank one projections in $\left\{P_{j}\right\}$ is easier than the general case, hence we present it separately. Recall a rank one projection $Q=|\xi\rangle\langle\xi|$ belongs to $\mathcal{A}^{\prime}=\operatorname{Fix}(\Phi)$ if and only if $\xi$ is a joint eigenvector for $A_{1}, \ldots, A_{n}$.

Lemma IV.6 Let $\left\{Q_{k}\right\}$ be the rank one projections from the set $\left\{P_{j}\right\}$. Let $Q_{k}=\left|\xi_{k}\right\rangle\left\langle\xi_{k}\right|$ and suppose $\lambda_{i k}$ are scalars with

$$
A_{i} \xi_{k}=\lambda_{i k} \xi_{k} \quad \text { for } \quad i=1, \ldots, n
$$

Define a function $f:\left\{Q_{k}\right\} \longrightarrow \mathbb{C}^{n}$ by

$$
f\left(Q_{k}\right)=\left(\lambda_{1 k}, \ldots, \lambda_{n k}\right)
$$

If $\lambda$ in $\mathbb{C}^{n}$ is such that $f^{-1}(\{\lambda\})$ is nonempty, then the projections in $f^{-1}(\{\lambda\})$ are linked in $\mathcal{A}$.

Proof. Let $f^{-1}(\{\lambda\})$ be a nonempty subset of $\left\{Q_{k}\right\}$. Observe that if $\xi_{k}$ and $\xi_{l}$ are range vectors for projections $Q_{k}$ and $Q_{l}$ in $f^{-1}(\{\lambda\})$, then they are eigenvectors for $\mathcal{A}$ and we have

$$
\left\langle A \xi_{k}|| \xi_{k}\right\rangle=\left\langle A \xi_{l} \| \xi_{l}\right\rangle \text { for all } A \in \mathcal{A}
$$

Indeed, $f\left(Q_{k}\right)=f\left(Q_{l}\right)$ and hence these inner products agree on a set of generators $A_{1}, \ldots, A_{n}$ for $\mathcal{A}$. Suppose $Q$ is a projection in $\mathcal{A}$ which is the sum of some, but not all, of the projections in $f^{-1}(\{\lambda\})$. Then there is a pair of vectors $\xi_{k}$ and $\xi_{l}$, each a range vector for a projection in $f^{-1}(\{\lambda\})$, such that $Q \xi_{k}=\xi_{k}$ and $Q \xi_{l}=0$. In particular, the identity (5) does not hold for $Q$, and consequently $Q$ does not belong to $\mathcal{A}$. 
For a nonempty set $f^{-1}(\{\lambda\})$, let $Q_{\lambda}$ be the sum of all projections inside $f^{-1}(\{\lambda\})$. It remains to show that each $Q_{\lambda}$ belongs to $\mathcal{A}$. From the structure theory for $\dagger$-algebras discussed at the start of this section, we know that for each $m$ the projection $\sum_{j \in \mathcal{S}_{m}} P_{j}$ belongs to $\mathcal{A}$, where $\mathcal{S}_{m}$ is the index set for all projections in $\left\{P_{j}\right\}$ of rank $m$. Let $P=$ $\sum_{j \in \mathcal{S}_{1}} P_{j}=\sum_{k}\left|\xi_{k}\right\rangle\left\langle\xi_{k}\right|$ be the projection obtained for $m=1$. Then it is easy to see that the compressions $A_{i} P=P A_{i}$, for $i=1, \ldots, n$, are mutually commuting operators inside $\mathcal{A}$ which are each diagonal with respect to the basis $\left\{\xi_{k}\right\}$ for the range of $P$. Hence it follows that $\mathcal{A} P$ is a subalgebra of $\mathcal{A}$ which, up to unitary equivalence, has a block matrix decomposition of the form

$$
\mathcal{A} P \simeq\left(\begin{array}{ccc}
a_{n_{1}} \mathbb{1}_{n_{1}} & & 0 \\
& \ddots & \\
0 & & a_{n_{d}} \mathbb{1}_{n_{d}}
\end{array}\right)
$$

Further, under this unitary equivalence, each projection onto a block $\mathbb{1}_{n_{j}}$ corresponds to a projection which is the sum of projections from a particular nonempty $f^{-1}(\{\lambda\})$. Since complete freedom is allowed for the scalars $a_{n_{j}}$, we see that every $Q_{\lambda}$ belongs to $\mathcal{A}$.

Remark IV.7 As a prelude to some of the examples below, we note that the case of an abelian algebra $\mathcal{A}$ corresponds to the case that the family of minimal $\mathcal{A}$-reducing projections are all rank one.

\section{Summary of the Algorithm}

We present a number of applications in the rest of the paper, but let us finish this section with a brief technical summary of the algorithm. Recall we have assumed that a set of vector space generators is known for $\operatorname{Fix}(\Phi)=\mathcal{A}^{\prime}=\operatorname{span}\left\{B_{1}, \ldots, B_{r}\right\}$.

\section{Part I.}

(i) If $\mathcal{A}^{\prime} \neq \mathbb{C} 1$, then choose $T=T^{\dagger}$ inside $\mathcal{A}^{\prime} \backslash \mathbb{C} 1$.

(ii) Compute the spectral projections $P_{1}, \ldots, P_{s}$ for $T$.

(iii) For each $P=P_{j}$, compute $P B_{1} P, \ldots, P B_{r} P$. If every $P B_{i} P$ belongs to $\mathbb{C} P$, then $P \mathcal{A}^{\prime} P=\mathbb{C} P$ and $P$ is a (non-zero) minimal reducing projection for $\mathcal{A}$, and it belongs to the maximal family. 
(iv) If some $B=B_{i}$ is such that $P B P$ does not belong to $\mathbb{C} P$, then $P$ is not minimal $\mathcal{A}$-reducing and the compression channel $\Phi_{P}$ has non-scalar fixed points; $P B P$ for instance. Then proceed through steps $(i),(i i)$ and $(i i i)$, perhaps a number of times, to find minimal $\mathcal{A}$-reducing mutually orthogonal projections supported on $P$.

\section{Part II.}

(i) Given the family $\left\{P_{j}\right\}$ from Part I, group together those projections with the same rank.

(ii) For the projections $\left\{P_{j, k}\right\}_{j \in \mathcal{S}_{k}}$ of rank $k \geq 2$, test for links between the $P_{j, k}$ by evaluating the commutators $\left[P_{\mathcal{S}}, B_{i}\right]$ where $P_{\mathcal{S}}=\sum_{j \in \mathcal{S}} P_{j, k}$ with $\mathcal{S} \subseteq \mathcal{S}_{k}$. Then $\left\{P_{j, k}: k \in \mathcal{S}\right\}$ are linked in $\mathcal{A}$ if and only if $\left[P_{\mathcal{S}}, B_{i}\right]=0$ for each $i$, and there is no proper subset of $\mathcal{S}$ with this property. The projections of rank $k=1$ may be tested using Lemma IV.6. The projections $P_{\mathcal{S}}$ defined by linked sets of projections will form the family of minimal central projections for $\mathcal{A}$.

(iii) The various links will give the $\dagger$-algebra structure of $\mathcal{A}$ up to unitary equivalence, and hence of $\mathcal{A}^{\prime}=\operatorname{Fix}(\Phi)$, along with the precise locations of matrix blocks inside $\mathcal{A}^{\prime}$.

\section{PHASE DAMPING AND RELATED EXAMPLES}

In specific cases the computations involved with the method described above can be less cumbersome than what is suggested. We begin with a number of simple illustrative applications of the process. In each of these cases the commutant is already well-known, or at least easy to find, but we shall demonstrate how to compute it using the algorithm. See [1, 14] for related examples.

Recall the Pauli matrices are given by

$$
\mathbb{1}_{2}=\left(\begin{array}{ll}
1 & 0 \\
0 & 1
\end{array}\right), X=\left(\begin{array}{ll}
0 & 1 \\
1 & 0
\end{array}\right), Y=\left(\begin{array}{cc}
0 & -i \\
i & 0
\end{array}\right), Z=\left(\begin{array}{cc}
1 & 0 \\
0 & -1
\end{array}\right) \text {. }
$$

We regard these as matrix representations of operators acting on $\mathbb{C}^{2}$ with respect to an orthonormal basis $\{|0\rangle,|1\rangle\}$ corresponding to the base states of a given two-level system. In the case of higher dimensions, for instance $\mathbb{C}^{4}$ with basis $\{|00\rangle,|01\rangle,|10\rangle,|11\rangle\}$, we use 
notation such as $Z_{1}$ and $Z_{2}$ to denote, respectively, the tensor products of $Z \otimes \mathbb{1}_{2}$ and $\mathbb{1}_{2} \otimes Z$ acting on $\mathbb{C}^{4}$.

Example V.1 Let $p$ be a positive real number, $0<p<1$. Let $A_{1}, A_{2}$ be operators on $\mathbb{C}^{2}$ defined on the standard basis by:

$$
A_{1}=(\sqrt{1-p}) \mathbb{1}_{2}=\sqrt{1-p}\left(\begin{array}{ll}
1 & 0 \\
0 & 1
\end{array}\right)
$$

and

$$
A_{2}=(\sqrt{p}) Z=\sqrt{p}\left(\begin{array}{cc}
1 & 0 \\
0 & -1
\end{array}\right) .
$$

Clearly $\sum_{k=1}^{2} A_{k} A_{k}^{\dagger}=\mathbb{1}_{2}=\sum_{k=1}^{2} A_{k}^{\dagger} A_{k}$, so $\left\{A_{1}, A_{2}\right\}$ are noise operators for a unital channel $\Phi$ acting on $\mathcal{B}\left(\mathbb{C}^{2}\right)$. The quantum operation corresponding to this channel is equivalent to the phase flip or phase damping operation on single qubits 21]. Its effect is to flip the phase of $|0\rangle$ relative to that of $|1\rangle$, and vice-versa, with probability $p$.

A simple computation shows that

$$
\begin{aligned}
\operatorname{Fix}(\Phi)=\mathcal{A}^{\prime} & =\left\{A_{1}, A_{2}\right\}^{\prime} \\
& =\left\{\left(\begin{array}{ll}
a & 0 \\
0 & b
\end{array}\right): a, b \in \mathbb{C}\right\} \simeq \mathbb{C} \mathbf{1} \oplus \mathbb{C} \mathbf{1} \\
& =\operatorname{Alg}\{Z\}=\mathcal{A} .
\end{aligned}
$$

Let us see how this structure arises through the algorithm. First choose a non-scalar operator in $\mathcal{A}^{\prime}$; in this case $Z$ will suffice. The rank one projections $P_{0}=|0\rangle\langle 0|$ and $P_{1}=|1\rangle\langle 1|$ are the spectral projections for $Z$, hence they are minimal inside $\mathcal{A}^{\prime}$ since the vectors $|0\rangle$ and |1) are joint eigenvectors for $A_{1}, A_{2}$. Thus the family $\left\{P_{0}, P_{1}\right\}$ forms the maximal family $\left(\mathbb{1}_{2}=P_{0}+P_{1}\right)$ of non-zero mutually orthogonal minimal projections inside the commutant $\mathcal{A}^{\prime}$.

Further observe that

$$
\left\{\begin{array} { l } 
{ A _ { 1 } | 0 \rangle = \sqrt { 1 - p } | 0 \rangle } \\
{ A _ { 2 } | 0 \rangle = \sqrt { p } | 0 \rangle }
\end{array} \quad \text { and } \quad \left\{\begin{array}{l}
A_{1}|1\rangle=\sqrt{1-p}|1\rangle \\
A_{2}|1\rangle=-\sqrt{p}|1\rangle
\end{array}\right.\right.
$$

The 2-tuples $(\sqrt{1-p}, \sqrt{p})$ and $(\sqrt{1-p},-\sqrt{p})$ are distinct, so the projections $P_{0}$ and $P_{1}$ are not linked by Lemma IV.6. Hence we have $\mathcal{A}=\mathcal{A} P_{0} \oplus \mathcal{A} P_{1}$, with $\mathcal{A} P_{0}$ and $\mathcal{A} P_{1}$ both 
subalgebras of $\mathcal{A}$ each unitarily equivalent to the one dimensional scalar algebra $\mathbb{C} 1$. Thus, the commutant $\mathcal{A}^{\prime}=\left(\mathcal{A} P_{0}\right)^{\prime}+\left(\mathcal{A} P_{1}\right)^{\prime}=P_{0} \mathcal{A}^{\prime} P_{0}+P_{1} \mathcal{A}^{\prime} P_{1}$ is also unitarily equivalent to $\mathbb{C} 1 \oplus \mathbb{C} 1$, and the form of $\operatorname{Fix}(\Phi)=\mathcal{A}^{\prime}$ given above is evident.

Example V.2 Let $p$ be a positive real number, $0<p<1$. Let $A_{1}, A_{2}$ be operators on $\mathbb{C}^{4}$ defined on the standard orthonormal basis by:

$$
A_{1}=\sqrt{1-p}\left(\mathbb{1}_{2} \otimes \mathbb{1}_{2}\right)=\sqrt{1-p}\left(\begin{array}{cccc}
1 & 0 & 0 & 0 \\
0 & 1 & 0 & 0 \\
0 & 0 & 1 & 0 \\
0 & 0 & 0 & 1
\end{array}\right)
$$

and

$$
A_{2}=\sqrt{p}\left(Z_{1} Z_{2}\right)=\sqrt{p}(Z \otimes Z)=\sqrt{p}\left(\begin{array}{cccc}
1 & 0 & 0 & 0 \\
0 & -1 & 0 & 0 \\
0 & 0 & -1 & 0 \\
0 & 0 & 0 & 1
\end{array}\right),
$$

Once again, it is clear that these noise operators determine a unital channel $\Phi$ on $\mathcal{B}\left(\mathbb{C}^{4}\right)$. We may compute

$$
\begin{aligned}
\operatorname{Fix}(\Phi)=\mathcal{A}^{\prime} & =\left\{A_{1}, A_{2}\right\}^{\prime} \\
& =\operatorname{Alg}\left\{Z_{1}, Z_{2}, X_{1} X_{2}+Y_{1} Y_{2}\right\} \\
& =\left\{\left(\begin{array}{cccc}
a_{11} & 0 & 0 & a_{14} \\
0 & a_{22} & a_{23} & 0 \\
0 & a_{32} & a_{33} & 0 \\
a_{41} & 0 & 0 & a_{44}
\end{array}\right): a_{i j} \in \mathbb{C}\right\} .
\end{aligned}
$$

By observation, we can see that $\mathcal{A}^{\prime}$ is unitarily equivalent to the orthogonal direct sum $\mathcal{A}^{\prime} \simeq \mathcal{M}_{2} \oplus \mathcal{M}_{2}$. To see this through the algorithm, first choose a non-scalar self-adjoint operator in $\operatorname{Fix}(\Phi)=\mathcal{A}^{\prime}$;

$$
Z_{1}=Z \otimes \mathbb{1}_{2}=\left(\begin{array}{cccc}
1 & 0 & 0 & 0 \\
0 & 1 & 0 & 0 \\
0 & 0 & -1 & 0 \\
0 & 0 & 0 & -1
\end{array}\right)=Z_{1}^{\dagger}
$$


is such an operator. The spectral projections for $Z_{1}$, corresponding to eigenvalues $\lambda=1$ and $\lambda=-1$, are given by

$$
P_{1}=|00\rangle\langle 00|+| 01\rangle\langle 01| \text { and } \quad P_{-1}=|10\rangle\langle 10|+| 11\rangle\langle 11| .
$$

Let $P_{k j}=|k j\rangle\langle k j|$ for $k, j \in\{0,1\}$. The projections $P_{1}$ and $P_{-1}$ belong to $\mathcal{A}^{\prime}$, but in this case they are not minimal inside $\mathcal{A}^{\prime}$. Indeed, observe that

$$
\begin{aligned}
P_{1} \mathcal{A}^{\prime} P_{1}= & \left(\mathcal{A} P_{1}\right)^{\prime}=\left\{A_{1} P_{1}, A_{2} P_{1}\right\}^{\prime} \\
= & \left\{\left(\begin{array}{cccc}
a_{11} & 0 & 0 & 0 \\
0 & a_{22} & 0 & 0 \\
0 & 0 & 0 & 0 \\
0 & 0 & 0 & 0
\end{array}\right): a_{11}, a_{22} \in \mathbb{C}\right\} \neq \mathbb{C} P_{1},
\end{aligned}
$$

and similarly for $P_{-1}$. Following the algorithm, we let

$$
Z_{1} Z_{2} P_{1}=P_{1} Z_{1} Z_{2}=\left(\begin{array}{cccc}
1 & 0 & 0 & 0 \\
0 & -1 & 0 & 0 \\
0 & 0 & 0 & 0 \\
0 & 0 & 0 & 0
\end{array}\right)
$$

be a non-scalar operator in $P_{1} \mathcal{A}^{\prime} P_{1}$. The spectral projections for this operator are

$$
P_{00}=|00\rangle\langle 00| \quad \text { and } \quad P_{01}=|01\rangle\langle 01| \text {. }
$$

These rank one projections are minimal inside $\mathcal{A}^{\prime}$. Similarly, we find the subprojections $P_{10}=|10\rangle\langle 10|$ and $P_{11}=|11\rangle\langle 11|$ of $P_{-1}$ are minimal inside $\mathcal{A}^{\prime}$, and hence the maximal family of non-zero mutually orthogonal minimal projections in $\mathcal{A}^{\prime}$ is given by

$$
\left\{P_{00}, P_{01}, P_{10}, P_{11}\right\}
$$

It remains to check for links between these minimal projections. Observe that

$$
\begin{aligned}
& \left\{\begin{array} { l l } 
{ A _ { 1 } | 0 0 \rangle } & { = \sqrt { 1 - p } | 0 0 \rangle } \\
{ A _ { 2 } | 0 0 \rangle } & { = \sqrt { p } | 0 0 \rangle }
\end{array} \quad \left\{\begin{array}{l}
A_{1}|01\rangle=\sqrt{1-p}|01\rangle \\
A_{2}|01\rangle=-\sqrt{p}|01\rangle
\end{array}\right.\right. \\
& \left\{\begin{array} { l } 
{ A _ { 1 } | 1 0 \rangle = \sqrt { 1 - p } | 1 0 \rangle } \\
{ A _ { 2 } | 1 0 \rangle = - \sqrt { p } | 1 0 \rangle }
\end{array} \quad \left\{\begin{array}{l}
A_{1}|11\rangle=\sqrt{1-p}|11\rangle \\
A_{2}|11\rangle=\sqrt{p}|11\rangle
\end{array}\right.\right.
\end{aligned}
$$


Thus, upon comparing the corresponding 2-tuples $(\sqrt{1-p}, \sqrt{p})$ and $(\sqrt{1-p},-\sqrt{p})$, we see that the pairs $\left\{P_{00}, P_{11}\right\}$ and $\left\{P_{01}, P_{10}\right\}$ are linked in $\mathcal{A}$ by Lemma IV.6. In particular, $P_{00}+P_{11}$ and $P_{01}+P_{10}$ belong to $\mathcal{A}=\mathcal{A}\left(P_{00}+P_{11}\right) \oplus \mathcal{A}\left(P_{01}+P_{10}\right)$ and $\mathcal{A}$ is unitarily equivalent to $\mathcal{A} \simeq \mathbb{C 1}_{2} \oplus \mathbb{C 1}_{2}$.

Hence, $\operatorname{Fix}(\Phi)=\mathcal{A}^{\prime} \simeq \mathcal{M}_{2} \oplus \mathcal{M}_{2}$, and the locations of the $\mathcal{M}_{2}$ blocks inside $\operatorname{Fix}(\Phi)=\mathcal{A}^{\prime}$ are explicitly given by the algorithm. Namely, one block of $2 \times 2$ matrices inside $\mathcal{A}^{\prime}=\operatorname{Fix}(\Phi)$ is given by all $T$ in $\mathcal{B}\left(\mathbb{C}^{4}\right)$ such that $T=\left(P_{00}+P_{11}\right) T\left(P_{00}+P_{11}\right)$, and similarly the other $\mathcal{M}_{2}$ block is supported on $P_{01}+P_{10}$. The blocks are disjoint in $\mathcal{A}^{\prime}$ in the sense that if $T$ belongs to $\mathcal{M}_{2}$, then an operator $\widetilde{T}=\left(P_{00}+P_{11}\right) T\left(P_{00}+P_{11}\right)$ can be defined in $\mathcal{A}^{\prime}$, and similarly for the $P_{01}+P_{10}$ block.

Example V.3 Let $p$ be a positive real number, $0<p<1$. Let $A_{1}, A_{2}, A_{3}, A_{4}$ be operators on $\mathbb{C}^{4}$ defined on the standard basis by:

$$
\begin{gathered}
A_{1}=(1-p)\left(\mathbb{1}_{2} \otimes \mathbb{1}_{2}\right)=(1-p)\left(\begin{array}{llll}
1 & 0 & 0 & 0 \\
0 & 1 & 0 & 0 \\
0 & 0 & 1 & 0 \\
0 & 0 & 0 & 1
\end{array}\right) \\
A_{2}=(\sqrt{p(1-p)}) Z_{1}=\sqrt{p(1-p)}\left(\begin{array}{cccc}
1 & 0 & 0 & 0 \\
0 & 1 & 0 & 0 \\
0 & 0 & -1 & 0 \\
0 & 0 & 0 & -1
\end{array}\right) \\
A_{3}=(\sqrt{p(1-p)}) Z_{2}=\sqrt{p(1-p)}\left(\begin{array}{cccc}
1 & 0 & 0 & 0 \\
0 & -1 & 0 & 0 \\
0 & 0 & 1 & 0 \\
0 & 0 & 0 & -1
\end{array}\right) \\
A_{4}=(p) Z_{1} Z_{2}=(p) Z \otimes Z=p\left(\begin{array}{cccc}
1 & 0 & 0 & 0 \\
0 & -1 & 0 & 0 \\
0 & 0 & -1 & 0 \\
0 & 0 & 0 & 1
\end{array}\right)
\end{gathered}
$$


These noise operators determine a unital channel $\Phi$ on $\mathcal{B}\left(\mathbb{C}^{4}\right)$. The fixed point algebra may be computed as

$$
\begin{aligned}
\operatorname{Fix}(\Phi)=\mathcal{A}^{\prime} & =\left\{A_{1}, A_{2}, A_{3}, A_{4}\right\}^{\prime}= \\
& =\left\{\left(\begin{array}{llll}
a & 0 & 0 & 0 \\
0 & b & 0 & 0 \\
0 & 0 & c & 0 \\
0 & 0 & 0 & d
\end{array}\right): a, b, c, d \in \mathbb{C}\right\} \\
& \simeq \mathbb{C} \mathbf{1} \oplus \mathbb{C} \mathbf{1} \oplus \mathbb{C} \mathbf{1} \oplus \mathbb{C} \mathbf{1}
\end{aligned}
$$

Running through the algorithm, we first choose $Z_{1}=Z \otimes \mathbb{1}_{2}=Z_{1}^{\dagger}$ as a non-scalar operator in $\mathcal{A}^{\prime}$. As in the previous example, the spectral projections for $Z_{1}$ are given by $P_{1}$ and $P_{-1}$. Once again, neither of these projections is minimal inside $\mathcal{A}^{\prime}$. For instance, for $P_{1}=|00\rangle\langle 00|+| 01\rangle\langle 01|$ the fixed point set of the restricted channel $\Phi_{P_{1}}(S)=\sum_{i=1}^{4} A_{k, 1} S A_{k, 1}^{\dagger}$, where $A_{k, 1}=A_{k} P_{1}$, satisfies

$$
\begin{aligned}
\operatorname{Fix}\left(\Phi_{P_{1}}\right)= & \left(\mathcal{A} P_{1}\right)^{\prime}=P_{1} \mathcal{A}^{\prime} P_{1} \\
= & \left\{\left(\begin{array}{llll}
a & 0 & 0 & 0 \\
0 & b & 0 & 0 \\
0 & 0 & 0 & 0 \\
0 & 0 & 0 & 0
\end{array}\right): a, b \in \mathbb{C}\right\} \neq \mathbb{C} P_{1},
\end{aligned}
$$

and hence contains non-scalar operators.

It follows that the projections $\left\{P_{00}, P_{01}, P_{10}, P_{11}\right\}$ again form the maximal family of nonzero mutually orthogonal minimal projections in $\operatorname{Fix}(\Phi)=\mathcal{A}^{\prime}$. But unlike the previous example, there are no links between these rank one projections. Indeed, observe that the numeric 4-tuples given by the equations

$$
\begin{aligned}
& \left\{\begin{array} { l } 
{ A _ { 1 } | 0 0 \rangle = ( 1 - p ) | 0 0 \rangle } \\
{ A _ { 2 } | 0 0 \rangle = \sqrt { p ( 1 - p ) } | 0 0 \rangle } \\
{ A _ { 3 } | 0 0 \rangle = \sqrt { p ( 1 - p ) } | 0 0 \rangle } \\
{ A _ { 4 } | 0 0 \rangle = p | 0 0 \rangle }
\end{array} \quad \left\{\begin{array}{l}
A_{1}|01\rangle=(1-p)|01\rangle \\
A_{2}|01\rangle=\sqrt{p(1-p)}|01\rangle \\
A_{3}|01\rangle=-\sqrt{p(1-p)}|01\rangle \\
A_{4}|01\rangle=-p|01\rangle
\end{array}\right.\right. \\
& \left\{\begin{array} { l } 
{ A _ { 1 } | 1 0 \rangle = ( 1 - p ) | 1 0 \rangle } \\
{ A _ { 2 } | 1 0 \rangle = - \sqrt { p ( 1 - p ) } | 1 0 \rangle } \\
{ A _ { 3 } | 1 0 \rangle = \sqrt { p ( 1 - p ) } | 1 0 \rangle } \\
{ A _ { 4 } | 1 0 \rangle = - p | 1 0 \rangle }
\end{array} \quad \left\{\begin{array}{l}
A_{1}|11\rangle=(1-p)|11\rangle \\
A_{2}|11\rangle=-\sqrt{p(1-p)}|11\rangle \\
A_{3}|11\rangle=-\sqrt{p(1-p)}|11\rangle \\
A_{4}|11\rangle=p|11\rangle
\end{array}\right.\right.
\end{aligned}
$$


are pairwise distinct. Thus each projection $P_{i j}$ belongs to $\mathcal{A}=\mathcal{A} P_{00}+\mathcal{A} P_{01}+\mathcal{A} P_{10}+\mathcal{A} P_{11}$, and $\mathcal{A}$ is unitarily equivalent to the unlinked orthogonal direct sum $\mathcal{A} \simeq \mathbb{C} \mathbf{1} \oplus \mathbb{C} \mathbf{1} \oplus \mathbb{C} 1 \oplus \mathbb{C} 1$. Hence, $\operatorname{Fix}(\Phi)=\mathcal{A}^{\prime} \simeq \mathbb{C} \mathbf{1} \oplus \mathbb{C} \mathbf{1} \oplus \mathbb{C} \mathbf{1} \oplus \mathbb{C} \mathbf{1}$ as well.

\section{UNITIZATION OF CHANNELS}

Before proceeding we present a discussion which will be useful in the sequel. Recall from the Spectral Theorem that every normal operator $T$ in $\mathcal{B}(\mathcal{H})$ decomposes as a sum $T=\lambda_{1} P_{1}+\ldots+\lambda_{r} P_{r}$ where $\lambda_{i}$ are the distinct eigenvalues for $T$ and $P_{i}$ the corresponding spectral projections. The functional calculus for $T$ is determined by all complex-valued functions $f$ which are defined on the eigenvalues of $T$. For each such function, there is an operator $f(T)$ in the functional calculus for $T$ defined by $f(T)=f\left(\lambda_{1}\right) P_{1}+\ldots+f\left(\lambda_{r}\right) P_{r}$.

Lemma VI.1 Let $T$ be a normal operator, and suppose $f$ is a function such that $f(T)$ belongs to the functional calculus for $T$. If $f$ is injective on the set of eigenvalues for $T$, then the commutant $\{T\}^{\prime}$ coincides with the commutant $\{f(T)\}^{\prime}$.

Proof. Suppose $T=\lambda_{1} P_{1}+\ldots+\lambda_{r} P_{r}$ is the spectral decomposition of $T$. Then, by the Spectral Theorem, the commutant of $T$ may also be realized as the commutant of its spectral projections; $\{T\}^{\prime}=\left\{P_{1}, \ldots, P_{r}\right\}^{\prime}$. By hypothesis, $f\left(\lambda_{i}\right)=f\left(\lambda_{j}\right)$ implies $i=j$. Thus, it follows that the spectral projections for $f(T)=f\left(\lambda_{1}\right) P_{1}+\ldots+f\left(\lambda_{r}\right) P_{r}$ are precisely $\left\{P_{1}, \ldots, P_{r}\right\}$. Hence, $\{T\}^{\prime}=\{f(T)\}^{\prime}$, as claimed.

As an immediate consequence of Theorem III.4, we obtain the following result for general quantum channels.

Corollary VI.2 If $\left\{A_{1}, \ldots, A_{n}\right\}$ are normal operators, and if, for each $k, f\left(A_{k}\right)$ is in the functional calculus of $A_{k}$ and is injective on the set of eigenvalues for $A_{k}$, then

$$
\left\{A_{1}, \ldots, A_{n}\right\}^{\prime}=\left\{f\left(A_{1}\right), \ldots, f\left(A_{n}\right)\right\}^{\prime}
$$

In particular, if

$$
\Phi(X)=\sum_{k=1}^{n} f\left(A_{k}\right) X f\left(A_{k}\right)^{\dagger}
$$

defines a unital quantum channel, then

$$
\operatorname{Fix}(\Phi)=\left\{A_{1}, \ldots, A_{n}\right\}^{\prime}
$$


Hence this gives a way of 'unitizing' a quantum channel, when the noise operators satisfy the hypotheses of Corollary VI.2, in such a way that the noise commutant of the new unital channel is completely determined by the commutant of the original noise operators. We shall make use of this lemma in the following section with the function $f$ given by a multiple of the natural exponential function.

\section{COLLECTIVE NOISE CHANNELS}

We next consider a class of examples which are more involved technically. The 3-qubit example from this class has been analyzed previously in the literature 10, 14, 16, 26] and also experimentally realized via nuclear magnetic resonance [27]. In particular, the algorithm allows us to explicitly identify the structure of the noise commutant for this channel. We will also conduct this analysis on the 4-qubit case, but first we outline the general $n$-qubit case.

Let $n \geq 3$ be a fixed positive integer. Let $\mathbf{X}$ be defined on $\mathbb{C}^{2^{n}}$ by

$$
\mathbf{X}=X_{1}+X_{2}+\ldots+X_{n}
$$

where

$$
X_{1}=X \otimes\left(\mathbb{1}_{2}^{\otimes(n-1)}\right), \quad X_{2}=\mathbb{1}_{2} \otimes X \otimes\left(\mathbb{1}_{2}^{\otimes(n-2)}\right), \ldots
$$

Similarly define $\mathbf{Y}=Y_{1}+\ldots+Y_{n}$ and $\mathbf{Z}=Z_{1}+\ldots+Z_{n}$. Observe that all of these operators are self-adjoint. Let $\exp (\cdot)$ be the complex-valued natural exponential function. This function belongs to the functional calculus of each of $\mathbf{X}, \mathbf{Y}, \mathbf{Z}$, and hence we may define operators $E_{x}, E_{y}, E_{z}$ on $\mathbb{C}^{2^{n}}$ by

$$
E_{x}=\frac{1}{\sqrt{3}} \exp (i \mathbf{X}), \quad E_{y}=\frac{1}{\sqrt{3}} \exp (i \mathbf{Y}), \quad E_{z}=\frac{1}{\sqrt{3}} \exp (i \mathbf{Z})
$$

A unital channel $\Phi$, called the $n$-qubit collective noise (rotation) channel, is then defined on $\mathcal{B}\left(\mathbb{C}^{2^{n}}\right)$ by

$$
\Phi(T)=E_{x} T E_{x}^{\dagger}+E_{y} T E_{y}^{\dagger}+E_{z} T E_{z}^{\dagger}
$$

As before, let $\mathcal{A}=\operatorname{Alg}\left\{E_{x}, E_{y}, E_{z}\right\}$ be the algebra generated by the noise operators. The following result provides a useful computational device for this class of channels. 
Proposition VII.1 If $\Phi$ is given by ([6), then

$$
\begin{aligned}
\operatorname{Fix}(\Phi)=\mathcal{A}^{\prime} & =\left\{E_{x}, E_{y}, E_{x}\right\}^{\prime}=\{\mathbf{X}, \mathbf{Y}, \mathbf{Z}\}^{\prime} \\
& =\{\mathbf{X}, \mathbf{Y}\}^{\prime}=\{\mathbf{X}, \mathbf{Z}\}^{\prime}=\{\mathbf{Y}, \mathbf{Z}\}^{\prime}
\end{aligned}
$$

Proof. The identities in the first line of this equation are a consequence of Theorem 11.4 and Corollary VI.2 for the equality $\left\{E_{x}, E_{y}, E_{x}\right\}^{\prime}=\{\mathbf{X}, \mathbf{Y}, \mathbf{Z}\}^{\prime}$ we use the fact that $\exp (i \cdot)$ is injective on the set of eigenvalues for each of $\mathbf{X}, \mathbf{Y}, \mathbf{Z}$. This can be seen by noting that the spectrum of $\mathbf{Z}$ consists of integers and that $\mathbf{X}, \mathbf{Y}, \mathbf{Z}$ are unitarily equivalent.

The equation $\{\mathbf{X}, \mathbf{Y}, \mathbf{Z}\}^{\prime}=\{\mathbf{X}, \mathbf{Y}\}^{\prime}$ follows from the anti-commutation relations for the Pauli matrices since

$$
\mathbf{X Y}-\mathbf{Y X}=\left(i \mathbf{Z}+\sum_{k \neq j} X_{k} Y_{j}\right)-\left(-i \mathbf{Z}+\sum_{k \neq j} Y_{j} X_{k}\right)=2 i \mathbf{Z} .
$$

The other equalities are similar.

Remark VII.2 Notice that we could also define a channel $\Phi$ for $n=1$ and $n=2$. But simple computations in both cases show that $\operatorname{Fix}(\Phi)=\{\mathbf{X}, \mathbf{Y}, \mathbf{Z}\}^{\prime}$ contains no bonafide matrix blocks; $\operatorname{Fix}(\Phi)=\mathbb{C}_{2}$ for $n=1$, and $\operatorname{Fix}(\Phi) \simeq \mathbb{C 1}_{3} \oplus \mathbb{C} 1$ for $n=2$. Hence these two channels are not of interest from a quantum computing perspective. On the other hand, there is rich structure in the commutant for $n \geq 3$.

\section{THE 3-QUBIT CASE}

In this section we consider the $n=3$ case for the collective noise channels. The matrix representations we use in this section are with respect to the standard ordered orthonormal basis for $\mathbb{C}^{8}$ associated with the usual tensor product notation $A \otimes B=\left(a_{i j} B\right)_{i j}$ for matrices. This is the basis

$$
\{|000\rangle,|001\rangle,|010\rangle,|011\rangle,|100\rangle,|101\rangle,|110\rangle,|111\rangle\} .
$$

We show explicitly that the algebra $\mathcal{A}$ is unitarily equivalent to the unlinked orthogonal direct sum of the full matrix algebra $\mathcal{M}_{4}$ together with the ampliation algebra $\mathcal{M}_{2} \otimes \mathbb{1}_{2}$;

$$
\mathcal{A} \simeq\left(\mathcal{M}_{2} \otimes \mathbb{1}_{2}\right) \oplus \mathcal{M}_{4}
$$


Thus the noise commutant contains an ampliated copy of $\mathcal{M}_{2}$;

$$
\begin{aligned}
& \operatorname{Fix}(\Phi)=\mathcal{A}^{\prime} \simeq\left(\mathcal{M}_{2} \otimes \mathbb{1}_{2}\right)^{\prime} \oplus\left(\mathcal{M}_{4}\right)^{\prime} \\
& \simeq\left(\mathbb{1}_{2} \otimes \mathcal{M}_{2}\right) \oplus \mathbb{C} \mathbb{1}_{4}
\end{aligned}
$$

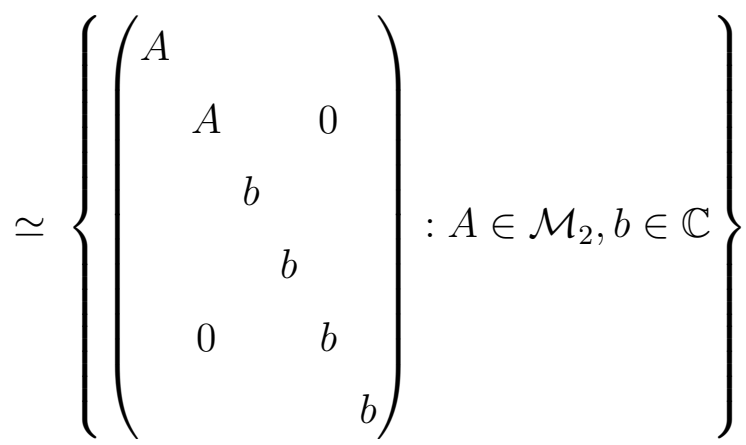

We will verify this by using the algorithm to produce the maximal family of non-zero minimal $\mathcal{A}$-reducing projections $\{P, Q, R\}$, and show that $P, Q$ are linked inside $\mathcal{A}$ with rank $P=$ $2=\operatorname{rank} Q$ and $\operatorname{rank} R=4$. In particular, this will imply that $\mathcal{A}=\mathcal{A}(P+Q) \oplus \mathcal{A} R$ with

$$
\mathcal{M}_{2} \otimes \mathbb{1}_{2} \simeq \mathcal{A}(P+Q) \subseteq \mathcal{A} \text { and } \quad \mathcal{M}_{4} \simeq \mathcal{A} R \subseteq \mathcal{A}
$$

and thus $\operatorname{Fix}(\Phi)=\mathcal{A}^{\prime}=(\mathcal{A}(P+Q))^{\prime} \oplus(\mathcal{A R})^{\prime}$ with

$$
\mathcal{M}_{2} \otimes \mathbb{1}_{2} \simeq(\mathcal{A}(P+Q))^{\prime} \subseteq \mathcal{A}^{\prime} \text { and } \quad \mathbb{C}_{1} \simeq(\mathcal{A} R)^{\prime} \subseteq \mathcal{A}^{\prime}
$$

We will also explicitly identify a copy of the Pauli matrices sitting inside the ampliation $\mathcal{M}_{2} \otimes \mathbb{1}_{2}$ contained in $\mathcal{A}^{\prime}=\operatorname{Fix}(\Phi)$.

We begin by computing $\mathcal{A}^{\prime}$ as a linear span. By Proposition VII.1 we have $\mathcal{A}^{\prime}=\{\mathbf{X}, \mathbf{Z}\}^{\prime}$. If we let $B=\left(b_{i j}\right)_{1 \leq i, j \leq 8}$ and consider the commutators $[B, \mathbf{Z}]$ and $[B, \mathbf{X}]$, we find a system of linear equations determined by the $b_{i j}$ which is satisfied precisely when $B$ belongs to $\mathcal{A}^{\prime}$. Specifically, the system includes 30 equations with a total of 20 unknowns. Thus we may apply basic linear algebra (we use Matlab to put the corresponding matrix in its reduced row echelon form) to obtain a set of vector space generators for $\mathcal{A}^{\prime}$. As it turns out, there are five parameters which yield matrices $B_{r}, B_{s}, B_{t}, B_{u}, B_{v}$. That is,

$$
\mathcal{A}^{\prime}=\operatorname{span}\left\{B_{r}, B_{s}, B_{t}, B_{u}, B_{v}\right\}
$$

But $B_{v}=B_{u}^{\dagger}$. Hence it is clear from Parts I and II that, for the purposes of the algorithm, we may restrict our attention to the set $\left\{B_{r}, B_{s}, B_{t}, B_{u}\right\}$. The matrix representations of 
these operators with respect to the standard basis for $\mathbb{C}^{8}$ are given below.

$$
\begin{aligned}
& B_{r}=B_{r}^{\dagger}=\left(\begin{array}{cccccccc}
1 & 0 & 0 & 0 & 0 & 0 & 0 & 0 \\
0 & 0 & 0 & 0 & 1 & 0 & 0 & 0 \\
0 & 0 & 0 & 0 & 1 & 0 & 0 & 0 \\
0 & 0 & 0 & -1 & 0 & 1 & 1 & 0 \\
0 & 1 & 1 & 0 & -1 & 0 & 0 & 0 \\
0 & 0 & 0 & 1 & 0 & 0 & 0 & 0 \\
0 & 0 & 0 & 1 & 0 & 0 & 0 & 0 \\
0 & 0 & 0 & 0 & 0 & 0 & 0 & 1
\end{array}\right) \\
& B_{s}=\left(\begin{array}{cccccccc}
0 & 0 & 0 & 0 & 0 & 0 & 0 & 0 \\
0 & 0 & 0 & 0 & 0 & 0 & 0 & 0 \\
0 & 0 & 1 & 0 & -1 & 0 & 0 & 0 \\
0 & 0 & 0 & 1 & 0 & -1 & 0 & 0 \\
0 & 0 & -1 & 0 & 1 & 0 & 0 & 0 \\
0 & 0 & 0 & -1 & 0 & 1 & 0 & 0 \\
0 & 0 & 0 & 0 & 0 & 0 & 0 & 0 \\
0 & 0 & 0 & 0 & 0 & 0 & 0 & 0
\end{array}\right) \\
& B_{t}=B_{t}^{\dagger}=\left(\begin{array}{cccccccc}
0 & 0 & 0 & 0 & 0 & 0 & 0 & 0 \\
0 & 1 & 0 & 0 & -1 & 0 & 0 & 0 \\
0 & 0 & 0 & 0 & 0 & 0 & 0 & 0 \\
0 & 0 & 0 & 1 & 0 & 0 & -1 & 0 \\
0 & -1 & 0 & 0 & 1 & 0 & 0 & 0 \\
0 & 0 & 0 & 0 & 0 & 0 & 0 & 0 \\
0 & 0 & 0 & -1 & 0 & 0 & 1 & 0 \\
0 & 0 & 0 & 0 & 0 & 0 & 0 & 0
\end{array}\right)
\end{aligned}
$$




$$
B_{u}=\left(\begin{array}{cccccccc}
0 & 0 & 0 & 0 & 0 & 0 & 0 & 0 \\
0 & 0 & 1 & 0 & -1 & 0 & 0 & 0 \\
0 & 0 & 0 & 0 & 0 & 0 & 0 & 0 \\
0 & 0 & 0 & 1 & 0 & -1 & 0 & 0 \\
0 & 0 & -1 & 0 & 1 & 0 & 0 & 0 \\
0 & 0 & 0 & 0 & 0 & 0 & 0 & 0 \\
0 & 0 & 0 & -1 & 0 & 1 & 0 & 0 \\
0 & 0 & 0 & 0 & 0 & 0 & 0 & 0
\end{array}\right)
$$

For Part I of the algorithm we shall conduct the spectral analysis on $B_{r}=B_{r}^{\dagger}$. The characteristic polynomial of $B_{r}$ may be computed as $\operatorname{det}\left(\lambda \mathbb{1}_{8}-B_{r}\right)=\lambda^{2}(\lambda-1)^{4}(\lambda+2)^{2}$. Let $P_{0}, P_{1}, P_{-2}$ be the projections onto the eigenspaces for the eigenvalues $\lambda=0, \lambda=1$, and $\lambda=-2$. These projections belong to $\mathcal{A}^{\prime}$ with $\operatorname{rank} P_{0}=2=\operatorname{rank} P_{-2}$ and $\operatorname{rank} P_{1}=4$. Define vectors $\left\{\xi_{0}, \eta_{0}, \xi_{-2}, \eta_{-2}\right\}$ in $\mathbb{C}^{8}$ as follows:

$$
\xi_{0}=\frac{1}{\sqrt{2}}\left(\begin{array}{c}
0 \\
1 \\
-1 \\
0 \\
0 \\
0 \\
0 \\
0
\end{array}\right) \eta_{0}=\frac{1}{\sqrt{2}}\left(\begin{array}{c}
0 \\
0 \\
0 \\
0 \\
1 \\
-1 \\
0
\end{array}\right) \xi_{-2}=\frac{1}{\sqrt{6}}\left(\begin{array}{c}
0 \\
1 \\
1 \\
-2 \\
0 \\
0 \\
0
\end{array}\right) \eta_{-2}=\frac{1}{\sqrt{6}}\left(\begin{array}{c}
0 \\
0 \\
0 \\
0 \\
-1 \\
-1 \\
0
\end{array}\right) .
$$

We note that these vectors are essentially the 'singlet-triplet' basis for the noiseless repre- 
sentation as given in [26] and also used in [7, 10, 14]. Then $P_{0}$ and $P_{-2}$ are given by

$$
\begin{aligned}
P_{0} & =\left|\xi_{0}\right\rangle\left\langle\xi_{0}|+| \eta_{0}\right\rangle\left\langle\eta_{0}\right| \\
& =\left(\begin{array}{cccccccc}
0 & 0 & 0 & 0 & 0 & 0 & 0 & 0 \\
0 & 1 / 2 & -1 / 2 & 0 & 0 & 0 & 0 & 0 \\
0 & -1 / 2 & 1 / 2 & 0 & 0 & 0 & 0 & 0 \\
0 & 0 & 0 & 0 & 0 & 0 & 0 & 0 \\
0 & 0 & 0 & 0 & 0 & 0 & 0 & 0 \\
0 & 0 & 0 & 0 & 0 & 1 / 2 & -1 / 2 & 0 \\
0 & 0 & 0 & 0 & 0 & -1 / 2 & 1 / 2 & 0 \\
0 & 0 & 0 & 0 & 0 & 0 & 0 & 0
\end{array}\right),
\end{aligned}
$$

and

$$
\begin{aligned}
P_{-2} & =\left|\xi_{-2}\right\rangle\left\langle\xi_{-2}|+| \eta_{-2}\right\rangle\left\langle\eta_{-2}\right| \\
& =\left(\begin{array}{cccccccc}
0 & 0 & 0 & 0 & 0 & 0 & 0 & 0 \\
0 & 1 / 6 & 1 / 6 & 0 & -1 / 3 & 0 & 0 & 0 \\
0 & 1 / 6 & 1 / 6 & 0 & -1 / 3 & 0 & 0 & 0 \\
0 & 0 & 0 & 2 / 3 & 0 & -1 / 3 & -1 / 3 & 0 \\
0 & -1 / 3 & -1 / 3 & 0 & 2 / 3 & 0 & 0 & 0 \\
0 & 0 & 0 & -1 / 3 & 0 & 1 / 6 & 1 / 6 & 0 \\
0 & 0 & 0 & -1 / 3 & 0 & 1 / 6 & 1 / 6 & 0 \\
0 & 0 & 0 & 0 & 0 & 0 & 0 & 0
\end{array}\right) .
\end{aligned}
$$

Furthermore, $P_{1}$ is computed as

$$
\begin{aligned}
P_{1} & =1_{8}-P_{0}-P_{-2} \\
& =\left(\begin{array}{ccccccccc}
1 & 0 & 0 & 0 & 0 & 0 & 0 & 0 \\
0 & 1 / 3 & 1 / 3 & 0 & 1 / 3 & 0 & 0 & 0 \\
0 & 1 / 3 & 1 / 3 & 0 & 1 / 3 & 0 & 0 & 0 \\
0 & 0 & 0 & 1 / 3 & 0 & 1 / 3 & 1 / 3 & 0 \\
0 & 1 / 3 & 1 / 3 & 0 & 1 / 3 & 0 & 0 & 0 \\
0 & 0 & 0 & 1 / 3 & 0 & 1 / 3 & 1 / 3 & 0 \\
0 & 0 & 0 & 1 / 3 & 0 & 1 / 3 & 1 / 3 & 0 \\
0 & 0 & 0 & 0 & 0 & 0 & 0 & 1
\end{array}\right) .
\end{aligned}
$$


Next we must examine the compressions of the commutant by these projections. First observe that $P_{\lambda} B_{r}=B_{r} P_{\lambda}=\lambda P_{\lambda}$ for $\lambda=-2,0,1$ as the $P_{\lambda}$ are spectral projections for $B_{r}$. We further compute

$$
\begin{gathered}
P_{-2} B_{s} P_{-2}=P_{-2} B_{t} P_{-2}=P_{-2} B_{u} P_{-2}=\left(\frac{3}{2}\right) P_{-2}, \\
P_{1} B_{s} P_{1}=P_{1} B_{t} P_{1}=P_{1} B_{u} P_{1}=0 \\
P_{0} B_{s} P_{0}=P_{0} B_{t} P_{0}=\left(\frac{1}{2}\right) P_{0} \quad \text { and } \quad P_{0} B_{u} P_{0}=\left(-\frac{1}{2}\right) P_{0} .
\end{gathered}
$$

Hence it follows that $P_{\lambda} \mathcal{A}^{\prime} P_{\lambda}=\mathbb{C} P_{\lambda}$ for $\lambda=-2,0,1$. Therefore, the minimal $\mathcal{A}$-reducing projections $\left\{P_{-2}, P_{0}, P_{1}\right\}$ form the desired set of projections.

It remains to check for links. Since linked projections have the same rank, the only possible link between these projections is $P_{-2}+P_{0}$. But notice that $P_{0} B_{u} \neq B_{u} P_{0}$, and hence

$$
P_{0} \notin\left(\mathcal{A}^{\prime}\right)^{\prime}=\mathcal{A}^{\prime \prime}=\mathcal{A} \text {. }
$$

Thus, it follows that $P_{0}$ and $P_{-2}$ must be linked inside $\mathcal{A}$. (One may check that $P_{0}+P_{-2} \in$ $\mathcal{A}=\mathcal{A}^{\prime \prime}$.) This shows that $\mathcal{A}$, and hence $\mathcal{A}^{\prime}$, has the form claimed above.

Finally, we shall exhibit a copy of the Pauli matrices inside $\mathcal{M}_{2} \otimes \mathbb{1}_{2} \simeq\left(P_{0}+P_{-2}\right) \mathcal{A}^{\prime}\left(P_{0}+\right.$ $\left.P_{-2}\right) \subseteq \mathcal{A}^{\prime}=\operatorname{Fix}(\Phi)$. We may accomplish this by first identifying a set of $2 \times 2$ matrix units $\left\{E_{11}, E_{12}, E_{21}, E_{22}\right\}$ inside $\left(P_{0}+P_{-2}\right) \mathcal{A}^{\prime}\left(P_{0}+P_{-2}\right)$. For this the algebra structure of $\mathcal{A}$ and $\mathcal{A}^{\prime}$ may be used together with a spectral analysis of the operators $\{\mathbf{X}, \mathbf{Y}, \mathbf{Z}\}$. Specifically, with the orthonormal basis $\left\{\xi_{0}, \eta_{0}, \xi_{-2}, \eta_{-2}\right\}$ for $P_{0} \mathbb{C}^{8} \oplus P_{-2} \mathbb{C}^{8}$ we may define

$$
\begin{aligned}
& E_{11}=\left|\xi_{0}\right\rangle\left\langle\xi_{0}|+| \eta_{0}\right\rangle\left\langle\eta_{0}\right|=P_{0} \\
& E_{12}=\left|\xi_{0}\right\rangle\left\langle\xi_{-2}|+| \eta_{0}\right\rangle\left\langle\eta_{-2}\right| \\
& E_{21}=\left|\xi_{-2}\right\rangle\left\langle\xi_{0}|+| \eta_{-2}\right\rangle\left\langle\eta_{0}\right| \\
& E_{22}=\left|\xi_{-2}\right\rangle\left\langle\xi_{-2}|+| \eta_{-2}\right\rangle\left\langle\eta_{-2}\right|=P_{-2}
\end{aligned}
$$

Then the matrix representations of operators inside $\left(P_{0}+P_{-2}\right) \mathcal{A}^{\prime}\left(P_{0}+P_{-2}\right)$ with respect to the basis $\left\{\xi_{0}, \xi_{-2}, \eta_{0}, \eta_{-2}\right\}$ (a 'canonical shuffle' [22, 23] of the above basis) have the form

$$
\left(P_{0}+P_{-2}\right) \mathcal{A}^{\prime}\left(P_{0}+P_{-2}\right) \simeq\left\{\left(\begin{array}{ll}
A & 0 \\
0 & A
\end{array}\right): A \in \mathcal{M}_{2}\right\},
$$


and the $E_{i j}$ are the natural matrix units in this decomposition. Thus, we may obtain versions of the Pauli matrices inside $\mathcal{A}^{\prime}=\operatorname{Fix}(\Phi)$ by defining

$$
\begin{gathered}
X=E_{12}+E_{21}=\frac{1}{\sqrt{3}}\left(\begin{array}{cccccccc}
0 & 0 & 0 & 0 & 0 & 0 & 0 & 0 \\
0 & 1 & 0 & 0 & -1 & 0 & 0 & 0 \\
0 & 0 & -1 & 0 & 1 & 0 & 0 & 0 \\
0 & 0 & 0 & 0 & 0 & 1 & -1 & 0 \\
0 & -1 & 1 & 0 & 0 & 0 & 0 & 0 \\
0 & 0 & 0 & 1 & 0 & -1 & 0 & 0 \\
0 & 0 & 0 & -1 & 0 & 0 & 1 & 0 \\
0 & 0 & 0 & 0 & 0 & 0 & 0 & 0
\end{array}\right) \\
Y=-i E_{12}+i E_{21}=\frac{1}{\sqrt{3}}\left(\begin{array}{cccccccc}
0 & 0 & 0 & 0 & 0 & 0 & 0 & 0 \\
0 & 0 & -1 & 0 & 1 & 0 & 0 & 0 \\
0 & 1 & 0 & 0 & -1 & 0 & 0 & 0 \\
0 & 0 & 0 & 0 & 0 & 1 & -1 & 0 \\
0 & -1 & 1 & 0 & 0 & 0 & 0 & 0 \\
0 & 0 & 0 & -1 & 0 & 0 & 1 & 0 \\
0 & 0 & 0 & 1 & 0 & -1 & 0 & 0 \\
0 & 0 & 0 & 0 & 0 & 0 & 0 & 0
\end{array}\right) \\
\left.0 \begin{array}{cccccccc}
0 \\
0 & 0 & 0 & 0 & 0 & 0 & 0 \\
0 & 1 & -2 & 0 & 1 & 0 & 0 & 0 \\
0 & -2 & 1 & 0 & 1 & 0 & 0 & 0 \\
0 & 0 & 0 & -2 & 0 & 1 & 1 & 0 \\
0 & 1 & 1 & 0 & -2 & 0 & 0 & 0 \\
0 & 0 & 0 & 1 & 0 & 1 & -2 & 0 \\
0 & 0 & 0 & 1 & 0 & -2 & 1 & 0 \\
0 & 0 & 0 & 0 & 0 & 0 & 0 & 0
\end{array}\right) .
\end{gathered}
$$

One may verify directly that the anti-commutation relations are satisfied by $\{X, Y, Z\}$, and that this triple belongs to $\operatorname{Fix}(\Phi)=\mathcal{A}^{\prime}=\{\mathbf{X}, \mathbf{Y}, \mathbf{Z}\}^{\prime}$. 


\section{THE 4-QUBIT CASE}

In this section we determine the structure of $\mathcal{A}^{\prime}=\operatorname{Fix}(\Phi)$ for the collective noise channel with $n=4$. We will show that $\mathcal{A}$ is unitarily equivalent to the unlinked orthogonal direct sum

$$
\mathcal{A} \simeq \mathbb{C} \mathbb{1}_{2} \oplus\left(\mathcal{M}_{3} \otimes \mathbb{1}_{3}\right) \oplus \mathcal{M}_{5}
$$

and hence

$$
\operatorname{Fix}(\Phi)=\mathcal{A}^{\prime} \simeq \mathcal{M}_{2} \oplus\left(\mathcal{M}_{3} \otimes \mathbb{1}_{3}\right) \oplus \mathbb{C} \mathbb{1}_{5}
$$

We can recognize a noiseless (decoherence-free) subspace [8, 20, 30] which supports the first summand in the decomposition of $\mathcal{A}$ and a noiseless subsystem in the second summand. We establish the above forms of $\mathcal{A}$ and $\mathcal{A}^{\prime}$ by identifying the maximal family of projections from Part I of the algorithm, $\left\{P_{-3}, P_{-1}, P_{0,-1 / 3}, P_{0,1}, P_{1}, P_{3}\right\}$, and show that: $P_{-3,} P_{1}$ are linked in $\mathcal{A}$ with rank $P_{k}=1$ for $k=-3,1$; the triple $P_{-1}, P_{0,-1 / 3}, P_{3}$ is linked in $\mathcal{A}$ with the rank of each equal to three; and $\operatorname{rank} P_{0,1}=5$.

As for the 3-qubit case we may use elementary linear algebra to identify a set of vector space generators for $\mathcal{A}^{\prime}$. It turns out that it is sufficient in this 4 -qubit case to obtain a set of self-adjoint generators which have real matrix entries. We find that $\mathcal{A}^{\prime}$ is spanned by 10 such operators. For succinctness we shall only display the generators used in the spectral analysis. Let

$$
B_{0}=B_{0}^{\dagger}=\left(\begin{array}{cccccccccccccccc}
0 & 0 & 0 & 0 & 0 & 0 & 0 & 0 & 0 & 0 & 0 & 0 & 0 & 0 & 0 & 0 \\
0 & 0 & 0 & 0 & 0 & 0 & 0 & 0 & 0 & 0 & 0 & 0 & 0 & 0 & 0 & 0 \\
0 & 0 & 0 & 0 & 1 & 0 & 0 & 0 & -1 & 0 & 0 & 0 & 0 & 0 & 0 & 0 \\
0 & 0 & 0 & 0 & 0 & 0 & 1 & 0 & 0 & 0 & -1 & 0 & 0 & 0 & 0 & 0 \\
0 & 0 & 1 & 0 & 0 & 0 & 0 & 0 & -1 & 0 & 0 & 0 & 0 & 0 & 0 & 0 \\
0 & 0 & 0 & 0 & 0 & 0 & 1 & 0 & 0 & 0 & 0 & 0 & -1 & 0 & 0 & 0 \\
0 & 0 & 0 & 1 & 0 & 1 & 0 & 0 & 0 & -2 & 0 & 0 & 0 & 0 & 0 & 0 \\
0 & 0 & 0 & 0 & 0 & 0 & 0 & 2 & 0 & 0 & 0 & -1 & 0 & -1 & 0 & 0 \\
0 & 0 & -1 & 0 & -1 & 0 & 0 & 0 & 2 & 0 & 0 & 0 & 0 & 0 & 0 & 0 \\
0 & 0 & 0 & 0 & 0 & 0 & -2 & 0 & 0 & 0 & 1 & 0 & 1 & 0 & 0 & 0 \\
0 & 0 & 0 & -1 & 0 & 0 & 0 & 0 & 0 & 1 & 0 & 0 & 0 & 0 & 0 & 0 \\
0 & 0 & 0 & 0 & 0 & 0 & 0 & -1 & 0 & 0 & 0 & 0 & 0 & 1 & 0 & 0 \\
0 & 0 & 0 & 0 & 0 & -1 & 0 & 0 & 0 & 1 & 0 & 0 & 0 & 0 & 0 & 0 \\
0 & 0 & 0 & 0 & 0 & 0 & 0 & -1 & 0 & 0 & 0 & 1 & 0 & 0 & 0 & 0 \\
0 & 0 & 0 & 0 & 0 & 0 & 0 & 0 & 0 & 0 & 0 & 0 & 0 & 0 & 0 & 0 \\
0 & 0 & 0 & 0 & 0 & 0 & 0 & 0 & 0 & 0 & 0 & 0 & 0 & 0 & 0 & 0
\end{array}\right) .
$$

This operator belongs to the commutant with characteristic polynomial $\operatorname{det}\left(\lambda \mathbb{1}_{16}-B_{0}\right)=$ $(\lambda+3)(\lambda+1)^{3} \lambda^{8}(\lambda-1)(\lambda-3)^{3}$. Consider the set of spectral projections $\left\{P_{-3}, P_{-1}, P_{0}, P_{1}, P_{3}\right\}$ corresponding to the eigenvalues $\lambda_{1}=-3, \lambda_{2}=-1, \lambda_{3}=0, \lambda_{4}=1, \lambda_{5}=3$. These projections belong to $\mathcal{A}^{\prime}$ with $\operatorname{rank} P_{-3}=1=\operatorname{rank} P_{1}$, $\operatorname{rank} P_{-1}=3=\operatorname{rank} P_{3}$, and $\operatorname{rank} P_{0}=8$. Let

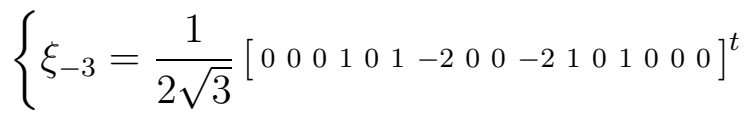




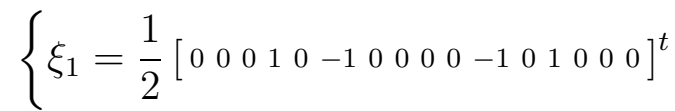

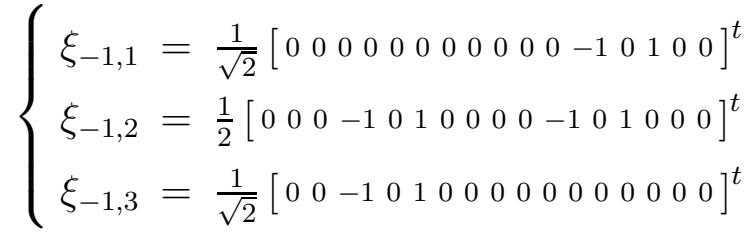

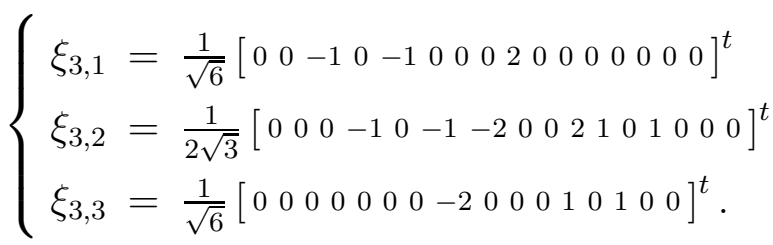

Then these projections may be computed as

$$
\begin{aligned}
P_{-3} & =\left|\xi_{1}\right\rangle\left\langle\xi_{1}\right| \\
P_{1} & =\left|\xi_{4}\right\rangle\left\langle\xi_{4}\right| \\
P_{-1} & =\left|\xi_{2,1}\right\rangle\left\langle\xi_{2,1}|+| \xi_{2,2}\right\rangle\left\langle\xi_{2,2}|+| \xi_{2,3}\right\rangle\left\langle\xi_{2,3}\right| \\
P_{3} & =\left|\xi_{5,1}\right\rangle\left\langle\xi_{5,1}|+| \xi_{5,2}\right\rangle\left\langle\xi_{5,2}|+| \xi_{5,3}\right\rangle\left\langle\xi_{5,3}\right| \\
P_{0} & =\mathbb{1}_{16}-P_{-3}-P_{-1}-P_{1}-P_{3}
\end{aligned}
$$

As rank one projections, $P_{-3}$ and $P_{1}$ are minimal in $\mathcal{A}^{\prime}$. Further, compressing the generators of $\mathcal{A}^{\prime}$ to $P_{-1}$ and $P_{3}$ reveals that $P \mathcal{A}^{\prime} P=\mathbb{C} P$ for both $P=P_{-1}$ and $P=P_{3}$. Hence $P_{-1}$ and $P_{3}$ belong to the maximal family. On the other hand, the projection $P_{0}$ is not minimal $\mathcal{A}$-reducing. There are a number of ways to see this, including verifying that $P_{0} \mathcal{A}^{\prime} P_{0} \neq \mathbb{C} P_{0}$ directly, but perhaps the easiest way is to observe that there are vector space generators for $\mathcal{A}^{\prime}$ which have all their eigenvalue multiplicities strictly less than $8=\operatorname{rank} P_{0}$. We could find the (unique) maximal family of projections by conducting a spectral analysis on any one of the non-scalar vector space generators of $\mathcal{A}^{\prime}$. Only two of the ten generators $B$ for $\mathcal{A}^{\prime}$ satisfy $P_{0} B P_{0} \neq 0$. They are:

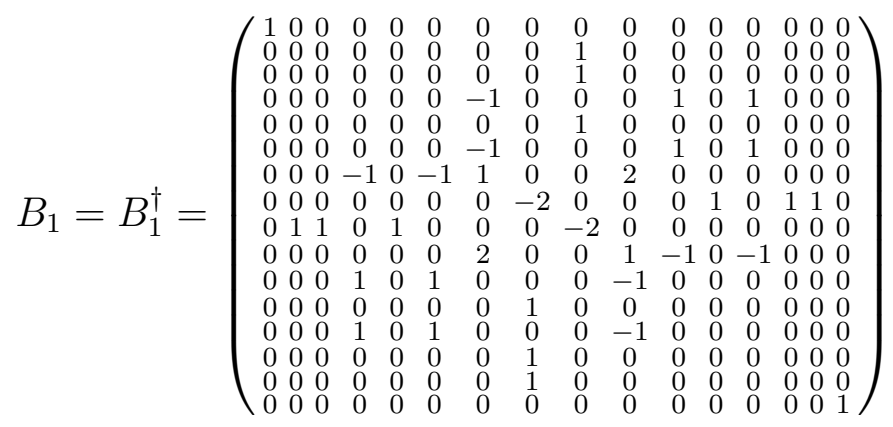


and

$$
B_{2}=B_{2}^{\dagger}=\left(\begin{array}{cccccccccccccccc}
0 & 0 & 0 & 0 & 0 & 0 & 0 & 0 & 0 & 0 & 0 & 0 & 0 & 0 & 0 & 0 \\
0 & 1 & 0 & 0 & 0 & 0 & 0 & 0 & -1 & 0 & 0 & 0 & 0 & 0 & 0 & 0 \\
0 & 0 & 0 & 0 & 0 & 0 & 0 & 0 & 0 & 0 & 0 & 0 & 0 & 0 & 0 & 0 \\
0 & 0 & 0 & 0 & 0 & 0 & 1 & 0 & 0 & 1 & -1 & 0 & -1 & 0 & 0 & 0 \\
0 & 0 & 0 & 0 & 0 & 0 & 0 & 0 & 0 & 0 & 0 & 0 & 0 & 0 & 0 & 0 \\
0 & 0 & 0 & 0 & 0 & 0 & 1 & 0 & 0 & 1 & -1 & 0 & -1 & 0 & 0 & 0 \\
0 & 0 & 0 & 1 & 0 & 1 & -2 & 0 & 0 & -2 & 1 & 0 & 1 & 0 & 0 & 0 \\
0 & 0 & 0 & 0 & 0 & 0 & 0 & 1 & 0 & 0 & 0 & 0 & 0 & 0 & -1 & 0 \\
0 & -1 & 0 & 0 & 0 & 0 & 0 & 0 & 1 & 0 & 0 & 0 & 0 & 0 & 0 & 0 \\
0 & 0 & 0 & 1 & 0 & 1 & -2 & 0 & 0 & -2 & 1 & 0 & 1 & 0 & 0 & 0 \\
0 & 0 & 0 & -1 & 0 & -1 & 1 & 0 & 0 & 1 & 0 & 0 & 0 & 0 & 0 & 0 \\
0 & 0 & 0 & 0 & 0 & 0 & 0 & 0 & 0 & 0 & 0 & 0 & 0 & 0 & 0 & 0 \\
0 & 0 & 0 & -1 & 0 & -1 & 1 & 0 & 0 & 1 & 0 & 0 & 0 & 0 & 0 & 0 \\
0 & 0 & 0 & 0 & 0 & 0 & 0 & 0 & 0 & 0 & 0 & 0 & 0 & 0 & 0 & 0 \\
0 & 0 & 0 & 0 & 0 & 0 & 0 & -1 & 0 & 0 & 0 & 0 & 0 & 0 & 1 & 0 \\
0 & 0 & 0 & 0 & 0 & 0 & 0 & 0 & 0 & 0 & 0 & 0 & 0 & 0 & 0 & 0
\end{array}\right) .
$$

Following the algorithm, we shall consider the spectral projections for $P_{0} B_{1} P_{0}$. We compute $\operatorname{det}\left(\lambda \mathbb{1}_{16}-P_{0} B_{1} P_{0}\right)=(\lambda+1 / 3)^{3} \lambda^{8}(\lambda-1)^{5}$. Let $P_{0,-1 / 3}, P_{0,0}, P_{0,1}$ be the projections onto the eigenspaces for $\lambda_{1}=-1 / 3, \lambda_{2}=0, \lambda_{3}=1$. Since $P_{0}^{\perp} \leq P_{0,0}$ and $\operatorname{rank} P_{0}^{\perp}=8=\operatorname{rank} P_{0,0}$, we have $P_{0,0}=P_{0}^{\perp}$, and we need only focus on $P_{0,-1 / 3}$ and $P_{0,1}$. Observe that rank $P_{0,-1 / 3}=3$ and $\operatorname{rank} P_{0,1}=5$. Let

$$
\begin{aligned}
& \eta_{1}=\frac{1}{2 \sqrt{3}}\left[\begin{array}{lllllllllllllll}
0 & 0 & 0 & 0 & 0 & 0 & 0 & 1 & 0 & 0 & 0 & 1 & 0 & 1 & -3
\end{array}\right]^{t} \\
& \eta_{2}=\frac{1}{\sqrt{6}}\left[\begin{array}{lllllllllllllll}
0 & 0 & 0 & -1 & 0 & -1 & 1 & 0 & 0 & -1 & 1 & 0 & 1 & 0 & 0
\end{array}\right]^{t}
\end{aligned}
$$

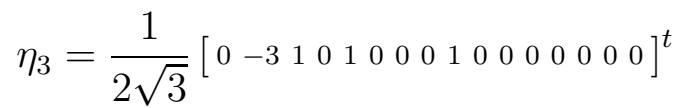

Then $P_{0,-1 / 3}$ and $P_{0,1}$ may be computed as

$$
\begin{aligned}
P_{0,-1 / 3} & =\left|\eta_{1}\right\rangle\left\langle\eta_{1}|+| \eta_{2}\right\rangle\left\langle\eta_{2}|+| \eta_{3}\right\rangle\left\langle\eta_{3}\right| \\
P_{0,1} & =P_{0}-P_{0,-1 / 3} .
\end{aligned}
$$

For the generators $B$ of $\mathcal{A}^{\prime}$ other than $B_{1}, B_{2}$ we have

$$
P_{0,-1 / 3}(B) P_{0,-1 / 3}=P_{0,-1 / 3}\left(P_{0} B P_{0}\right) P_{0,-1 / 3}=0,
$$

and similarly $P_{0,1} B P_{0,1}=0$. Moreover,

$$
P_{0,-1 / 3}\left(B_{1}\right) P_{0,-1 / 3}=-\frac{1}{3} P_{0,-1 / 3} \quad P_{0,-1 / 3}\left(B_{2}\right) P_{0,-1 / 3}=\frac{4}{3} P_{0,-1 / 3}
$$

and

$$
P_{0,1}\left(B_{1}\right) P_{0,1}=P_{0,1} \quad P_{0,1}\left(B_{2}\right) P_{0,1}=0 .
$$

Thus $P \mathcal{A}^{\prime} P=\mathbb{C} P$ for both $P=P_{0,-1 / 3}$ and $P=P_{0,1}$, and hence $P_{0,-1 / 3}$ and $P_{0,1}$ are minimal $\mathcal{A}$-reducing projections. 
As $\mathbb{1}_{16}=P_{-3}+P_{-1}+P_{0,-1 / 3}+P_{0,1}+P_{1}+P_{3}$, the set of minimal $\mathcal{A}$-reducing projections $\left\{P_{-3}, P_{-1}, P_{0,-1 / 3}, P_{0,1}, P_{1}, P_{3}\right\}$ does indeed form the maximal family of projections we seek. It remains to find links. The commutators $\left[P_{-3}, B\right]$ for generators $B$ of $\mathcal{A}^{\prime}$ are not all zero. Hence, as the only rank one projections in this family, we may deduce that $P_{-3}$ and $P_{1}$ are linked inside $\mathcal{A}$. Thus, $P_{-3}+P_{1} \in\left(\mathcal{A}^{\prime}\right)^{\prime}=\mathcal{A}$ and the block $\mathcal{A}\left(P_{-3}+P_{1}\right)=\left(P_{-3}+P_{1}\right) \mathcal{A} \simeq \mathbb{C} \mathbb{1}_{2}$ so that

$$
\mathcal{A}^{\prime}\left(P_{-3}+P_{1}\right)=\left(P_{-3}+P_{1}\right) \mathcal{A}^{\prime} \simeq \mathcal{M}_{2}
$$

Further, a check of commutators $[P, B]$ for generators $B$ of $\mathcal{A}^{\prime}$ with $P=P_{-1}, P_{3}, P_{0-1 / 3}$ shows that none of these projections belongs to $\left(\mathcal{A}^{\prime}\right)^{\prime}=\mathcal{A}$. It follows that $P_{-1}, P_{3}, P_{0,-1 / 3}$ are linked inside $\mathcal{A}$, and thus

$$
\begin{aligned}
\mathcal{A}\left(P_{-1}+P_{3}+P_{0,-1 / 3}\right) & \simeq \mathcal{M}_{3} \otimes \mathbb{1}_{3} \\
& \simeq \mathbb{1}_{3} \otimes \mathcal{M}_{3} \\
& \simeq\left(\mathcal{M}_{3} \otimes \mathbb{1}_{3}\right)^{\prime} \simeq \mathcal{A}^{\prime}\left(P_{-1}+P_{3}+P_{0,-1 / 3}\right) .
\end{aligned}
$$

Finally, we have $\mathcal{A} P_{0,1} \simeq \mathcal{M}_{5}$ and $\mathcal{A}^{\prime} P_{0,1} \simeq\left(\mathcal{M}_{5}\right)^{\prime}=\mathbb{C}_{5}$, and the structure of $\operatorname{Fix}(\Phi)=\mathcal{A}^{\prime}$ is now apparent.

We finish by identifying copies of the Pauli matrices belonging to the matrix blocks inside $\mathcal{A}^{\prime}=\operatorname{Fix}(\Phi)$. For the block $\mathcal{M}_{2} \simeq \mathcal{A}^{\prime}\left(P_{-3}+P_{1}\right)$, we may define matrix units in $\mathcal{A}^{\prime}$ by

$$
\begin{array}{ll}
E_{11}=P_{-3}=\left|\xi_{-3}\right\rangle\left\langle\xi_{-3}\right| & E_{12}=\left|\xi_{-3}\right\rangle\left\langle\xi_{1}\right| \\
E_{21}=\left|\xi_{1}\right\rangle\left\langle\xi_{-3}\right| & E_{22}=P_{1}=\left|\xi_{1}\right\rangle\left\langle\xi_{1}\right|
\end{array}
$$

In particular, we may define the following operators inside $\mathcal{A}^{\prime}\left(P_{-3}+P_{1}\right) \subseteq \mathcal{A}^{\prime}=\operatorname{Fix}(\Phi)$ :

$$
X=E_{12}+E_{21}, \quad Y=-i E_{12}+i E_{21}, \quad Z=E_{11}-E_{22} .
$$

We may also obtain matrix units inside $\mathcal{A}^{\prime}$ for the copy of $\mathcal{M}_{3} \otimes \mathbb{1}_{3} \simeq \mathcal{A}^{\prime}\left(P_{-1}+P_{3}+P_{0,-1 / 3}\right) \subseteq$ $\mathcal{A}^{\prime}$. For instance, a set of $2 \times 2$ matrix units inside this block is given by:

$$
\begin{array}{ll}
F_{11}=P_{-1}=\sum_{k=1}^{3}\left|\xi_{-1, k}\right\rangle\left\langle\xi_{-1, k}\right| & F_{12}=\sum_{k=1}^{3}\left|\xi_{-1, k}\right\rangle\left\langle\xi_{3, k}\right| \\
F_{21}=\sum_{k=1}^{3}\left|\xi_{3, k}\right\rangle\left\langle\xi_{-1, k}\right| & F_{22}=P_{3}=\sum_{k=1}^{3}\left|\xi_{3, k}\right\rangle\left\langle\xi_{3, k}\right|
\end{array}
$$

Thus, a second copy of the Pauli matrices inside $\mathcal{A}^{\prime}=\operatorname{Fix}(\Phi)$ is realized by defining

$$
X=F_{12}+F_{21}, \quad Y=-i F_{12}+i F_{21}, \quad Z=F_{11}-F_{22} .
$$




\section{CONCLUSION}

Given a unital quantum channel $\Phi$, we have derived a constructive proof for computing the explicit algebra structure, as in (44), of the associated interaction algebra $\mathcal{A}$ and noise commutant $\mathcal{A}^{\prime}$. We mention that Zarikian [31] has recently written Matlab algorithms which also utilizes much of the theory discussed here to compute, among other things, the structures of such operator algebras. An important subtlety in this process involves the detection of ampliations within these algebras which arise from linked minimal projections. In the case of non-unital noise the algorithm may be easily adapted to find the structures of $\mathcal{A}$ and $\mathcal{A}^{\prime}$, since both are $\dagger$-algebras by definition, but the connection with the fixed point set $\operatorname{Fix}(\Phi)=\mathcal{A}^{\prime}$ is no longer valid.

As illustrations we worked through the process for several simple channels and the $n=3$ and $n=4$ qubit cases of the collective noise channels which arise from collective rotations. We have also used our method to compute the noise commutant for the 5 -qubit case, it is given by $\mathcal{A}^{\prime} \simeq \mathbb{C}_{6} \oplus\left(\mathcal{M}_{4} \otimes \mathbb{1}_{4}\right) \oplus\left(\mathcal{M}_{5} \otimes \mathbb{1}_{2}\right)$.

While the algorithm is capable of analyzing any situation, in particular cases a more specialized and conceptual investigation may be necessary. For instance, when $n>>0$ the computations in the algorithm required to compute the noise commutant of the $n$-qubit collective noise channel make the problem infeasible. In forthcoming work, the authors and

Poulin 12] use such a conceptual investigation to derive the explicit structure of the noise commutant for the general $n$-qubit case of the collective noise channels arising from collective rotation.

Acknowledgements. We thank the referee for several helpful comments. We are grateful to the Perimeter Institute for providing resources which helped foster this collaboration. We also acknowledge funding from NSERC, MITACS, and ARDA. The second author would like to thank Michele Mosca of the Institute for Quantum Computing and members of the Department of Mathematics at Purdue University for kind hospitality during recent visits.

[1] D. Aharonov, M. Ben-Or. Fault-tolerant quantum computation with constant error, In Proc. 29th. Ann. ACM Symp. on Theory of Computing, page 176, New York, 1998, ACM. quant-ph/9906129, quant-ph/9611025. 
[2] W. Arveson, An invitation to $\mathrm{C}^{*}$-algebras, Graduate Texts in Mathematics, No. 39, SpringerVerlag, New York-Heidelberg, 1976.

[3] O. Bratteli, P.E.T. Jorgensen, A. Kishimoto, R.F. Werner, Pure States on $\mathcal{O}_{d}$, J. Operator Theory, 43(2000), 97-143.

[4] M.D. Choi, Completely positive linear maps on complex matrices, Lin. Alg. Appl. 10 (1975), 285-290.

[5] K.R. Davidson, C*-algebras by example, Fields Institute Monographs, 6, Amer. Math. Soc., Providence, 1996.

[6] K.R. Davidson, D.W. Kribs, M.E. Shpigel, Isometric dilations of non-commuting finite rank n-tuples, Can. J. Math. 53 (2001), 506-545.

[7] D.P. DiVincenzo, D. Bacon, J. Kempe, G. Burkard, K.B. Whaley, Universal quantum computation with the exchange interaction, Nature 408, 3392000.

[8] L.-M. Duan, G.-C. Guo, Preserving coherence in quantum computation by pairing quantum bits, Phys. Rev. Lett. 79 (1997), 1953.

[9] S. De Filippo, Quantum computation using decoherence-free states of the physical operator algebra, Phys. Rev. A 62, 052307 (2000).

[10] E.M. Fortunato, L. Viola, M.A. Pravia, E. Knill, R. Laflamme, T.F. Havel, D.G. Cory, Exploring noiseless subsystems via nuclear magnetic resonance, Phys. Rev. A 67, 062303 (2003), quant-ph/0210057.

[11] E.M. Fortunato, L. Viola, J. Hodges, G. Teklemariam, D.G. Cory, Implementation of universal control on a decoherence-free qubit, New J. Phys. 4, 5.1 (2002).

[12] J.A. Holbrook, D.W. Kribs, R. Laflamme, D. Poulin, Noiseless subsystems for collective rotation channels in quantum information theory, preprint, 2003.

[13] P.E.T. Jorgensen, Minimality of the data in wavelet filters, Adv. in Math., 159 (2001), 143228.

[14] J. Kempe, D. Bacon, D.A. Lidar, K.B. Whaley, Theory of decoherence-free fault-tolerant universal quantum computation, Phys. Rev. A 63, 042307 (2001).

[15] A. Yu. Kitaev, Quantum error correction with imperfect gates, in O. Hirota et al., editor, Quantum Communication and Computing and Measurement, New York, 1997.

[16] E. Knill, R. Laflamme, L. Viola, Theory of quantum error correction for general noise, Phys. Rev. Lett. 84, 2525 (2000). 
[17] E. Knill, R. Laflamme, W. H. Zurek, Resilient quantum computation: error models and thresholds, Science 279, 342 (1998).

[18] K. Kraus, General state changes in quantum theory, Ann. Physics 64 (1971), 311-335.

[19] D.W. Kribs, Quantum channels, wavelets, dilations, and representations of $\mathcal{O}_{n}$, Proc. Edin. Math. Soc., 46 (2003), 421-433.

[20] D. A. Lidar, I. L. Chuang, K. B. Whaley, Decoherence free subspaces for quantum computation, Phys. Rev. Lett. 81, 2594 (1998).

[21] M.A. Nielsen, I.L. Chuang, Quantum computation and quantum information, Cambridge University Press, 2000.

[22] V. Paulsen, Completely bounded maps and dilations, Pitman Res. Notes Math. 146, Longman Sci. Tech. Harlow, 1986.

[23] V. Paulsen, Completely bounded maps and operator algebras, Cambridge University Press, Cambridge, United Kingdom, 2002.

[24] J. Preskill, Reliable quantum computers, Proc. R. Soc. Lond. A, 454 (1998), 385-410.

[25] M. Takesaki, Theory of operator algebras I, Springer-Verlag, New York-Heidelberg, 1979.

[26] L. Viola, E. Knill, R. Laflamme, Constructing qubits in physical systems, J. Phys. A 34, 7067 (2001).

[27] L. Viola, E.M. Fortunato, M.A. Pravia, E. Knill, R. Laflamme, D.G. Cory, Experimental realization of noiseless subsystems for quantum information processing, Science 293, 2059 (2001).

[28] P. Zanardi, S. Lloyd, Topological protection and quantum noiseless subsystems, Phys. Rev. Lett. 90, 067902 (2003).

[29] P. Zanardi, Stabilizing quantum information, Phys. Rev. A 63, 012301 (2001).

[30] P. Zanardi, M. Rasetti, Noiseless quantum codes, Phys. Rev. Lett. 79, 3306 (1997).

[31] V. Zarikian, Algorithms for operator algebra calculations, preprint, 2003. 